\title{
Hadronic production of bottom-squark pairs with electroweak contributions
}

\author{
Jan Germer, ${ }^{a}$ Wolfgang Hollik ${ }^{a}$ and Edoardo Mirabella ${ }^{b}$ \\ ${ }^{a}$ Max-Planck-Institut für Physik, \\ Föhringer Ring 6, D-80805 München, Germany \\ ${ }^{b}$ Institut de Physique Théorique, CEA-Saclay, \\ F-91191, Gif-sur-Yvette cedex, France \\ E-mail: germer@mppmu.mpg.de, hollik@mppmu.mpg.de, \\ edoardo.mirabella@cea.fr
}

ABSTRACT: We present the complete computation of the tree-level and the next-to-leading order electroweak contributions to bottom-squark pair production at the LHC. The computation is performed within the minimal supersymmetric extension of the Standard Model. We discuss the numerical impact of these contributions in several supersymmetric scenarios.

KeYwords: Supersymmetric Standard Model, NLO Computations, Hadronic Colliders 


\section{Contents}

$\begin{array}{llr}1 & \text { Introduction } & 1\end{array}$

2 Tree-level cross section 3

2.1 Tree-level QCD contributions 3

2.2 Tree-level EW contributions 4

3 Next-to-leading order EW contributions 4

3.1 Virtual corrections 5

$\begin{array}{lll}3.2 & \text { Real corrections } & 6\end{array}$

$\begin{array}{lll}3.3 & \text { Resummation in the } \mathrm{b} / \tilde{\mathrm{b}} \text { sector } & 7\end{array}$

4 Numerical results $\quad 8$

4.1 Input parameters 8

4.2 Total hadronic cross section $\quad 9$

$\begin{array}{lll}4.3 & \text { Parameter scan } & 11\end{array}$

$\begin{array}{lll}4.4 & \text { Differential distributions } & 14\end{array}$

5 Other processes leading to bottom-squark pair production $\quad 17$

6 Conclusions 20

$\begin{array}{ll}\text { A Renormalization of the stop and sbottom sector } & 21\end{array}$

$\begin{array}{ll}\text { B Feynman diagrams } & 24\end{array}$

\section{Introduction}

Supersymmetry (SUSY) is a well motivated Beyond the Standard Model (BSM) scenario. Electroweak precision data indicates that SUSY should be realized at the TeV scale or below. If this is the case, it will be accessible to direct experimental measurements at the Large Hadron Collider (LHC) through the production of SUSY particles. Monte Carlo simulations have shown the possibility of the discovery of TeV-scale SUSY with $1 \mathrm{fb}^{-1}$ of integrated luminosity [1, 2]. First measurements involving supersymmetry-sensitive variables have been performed by both ATLAS [3-6] and CMS [7] collaborations.

At the LHC, colored particles like the SUSY partners of quarks and gluons, i.e. squarks and gluinos, will be copiously produced. Theoretically, these processes are extensively studied within the Minimal Supersymmetric extension of the Standard Model (MSSM). The leading-order (LO) contributions, of $\mathcal{O}\left(\alpha_{s}\right)$, have been known for a long time [8-12]. The 
next-to leading order (NLO) QCD corrections have been computed [13, 14] and implemented in the public code PROSPINO [15]. They affect the LO predictions substantially and they reduce the scale dependence considerably. More recent is the estimation of the logarithmically enhanced next-to-next-to-leading order (NNLO) QCD contributions to squark hadroproduction, the resummation of the QCD Sudakov logarithms at the nextto-leading-logarithmic (NLL) accuracy, and the resummation of the leading Coulomb corrections [16-22]. Their contribution amounts up to $10 \%$ for squark and gluino masses of the order of $1 \mathrm{TeV}$. They further stabilize the prediction against scale variation.

The electroweak (EW) contributions have been computed for several processes producing colored SUSY particles. They exhibit an extremely rich and complicate pattern, in particular when the EW contributions appear already at tree level. The tree-level EW contributions are of $\mathcal{O}\left(\alpha^{2}+\alpha_{s} \alpha\right)$. At the parton level, they arise from $q \bar{q}$-annihilation, $q q$-scattering or photon-induced processes, depending on the final state considered. They are known [23-28] and they can increase the LO cross section by up to $20 \%$. The impact of these contributions in the context of non-minimal flavor violation and explicit $\mathrm{CP}$ violation has been investigated as well $[29,30]$. The NLO EW corrections contribute at $\mathcal{O}\left(\alpha_{s}^{2} \alpha\right)$ and have been computed for stop-anti-stop [23, 31], squark-(anti-)squark [28, 32], gluino-squark [25], and gluino-gluino [33] production. Their size is comparable with that of the tree-level EW and NNLO QCD contributions, and their impact strongly depend on the SUSY scenario considered.

The production of third generation squarks is special. The non-negligible mixing in the stop and sbottom sector could lead to relatively low masses for the lightest bottom and top squarks, favoring their direct production at the LHC. Moreover $b$-tagging makes bottom- and top-squark production experimentally distinguishable from the production of the squarks of the first two generations [34-36]. A dedicated analysis looking for third generation squark production at the LHC is already available [6]. This kind of searches are particularly important in SUSY scenarios such as the ATLAS benchmark scenario SU6, where inclusive searches with jets, missing transverse energy, and leptons are problematic [2].

In this paper we focus on the hadronic production of bottom-squark pairs

$$
P P \rightarrow \tilde{b}_{\alpha} \tilde{b}_{\beta}^{*}, \tilde{b}_{\alpha} \tilde{b}_{\beta}, \tilde{b}_{\alpha}^{*} \tilde{b}_{\beta}^{*}, \quad \alpha, \beta \in\{1,2\} .
$$

In particular we present the first complete computation of the NLO EW corrections to diagonal sbottom-anti-sbottom pair production,

$$
P P \rightarrow \tilde{b}_{\alpha} \tilde{b}_{\alpha}^{*}, \quad \alpha \in\{1,2\}
$$

The contribution of the remaining processes is small (cf. section 5), hence we will not include them in our discussion on the EW corrections. The process (1.2) exhibits specific features like the mixing between left- and right-handed bottom squarks, the renormalization of the sbottom sector $[37,38]$, the non-negligible Higgs-boson contributions, and the enhanced Yukawa couplings for large values of $\tan \beta$ with the related need of resummation [39]. These features make the computations of the electroweak contributions to the processes (1.2) substantially different from those for squark-anti-squark [32] and stop-antistop [23] production, and justify a specific investigation, which is reported in this paper. 
The outline of the paper is as follows. In section 2 we summarize the tree-level contributions to the processes (1.2). Section 3 describes the various partonic processes contributing at $\mathcal{O}\left(\alpha_{s}^{2} \alpha\right)$ and the strategy of the calculation. The numerical impact of the NLO EW contributions at the LHC with $\sqrt{S}=14 \mathrm{TeV}$ and $\sqrt{S}=7 \mathrm{TeV}$ is presented in section 4. In section 5 we discuss the numerical impact of the subleading bottom-squark pair production processes. The Feynman diagrams and the technical details of the renormalization of the sbottom sector are collected in the appendix.

\section{Tree-level cross section}

In this section we describe the tree-level contributions to the process (1.2), which are of order $\mathcal{O}\left(\alpha_{s}^{2}\right), \mathcal{O}\left(\alpha_{s} \alpha\right)$, and $\mathcal{O}\left(\alpha^{2}\right)$. We will conventionally denote the cross section (amplitude) of a partonic process $X$ at a given order $\mathcal{O}\left(\alpha_{s}^{a} \alpha^{b}\right)$ as $\mathrm{d} \hat{\sigma}_{X}^{a, b}\left(\mathcal{M}_{X}^{a, b}\right)$. The parton luminosities are defined as

$$
\frac{\mathrm{d} L_{i j}}{\mathrm{~d} \tau}(\tau)=\frac{1}{1+\delta_{i j}} \int_{\tau}^{1} \frac{\mathrm{d} x}{x}\left[f_{i}\left(\frac{\tau}{x}, \mu_{F}\right) f_{j}\left(x, \mu_{F}\right)+f_{j}\left(x, \mu_{F}\right) f_{i}\left(\frac{\tau}{x}, \mu_{F}\right)\right],
$$

where $f_{i}$ is the parton distribution function (PDF) of the parton $i$ inside the proton.

\subsection{Tree-level QCD contributions}

The leading-order cross section, of the order $\mathcal{O}\left(\alpha_{s}^{2}\right)$, is given by

$$
\begin{aligned}
\mathrm{d} \sigma_{P P \rightarrow \tilde{b}_{\alpha} \tilde{b}_{\alpha}^{*}}^{\mathrm{LOQD}}(S)= & \int_{\tau_{0}}^{1} \mathrm{~d} \tau \frac{\mathrm{d} L_{g g}}{\mathrm{~d} \tau} \mathrm{d} \hat{\sigma}_{g g \rightarrow \tilde{b}_{\alpha} \tilde{b}_{\alpha}^{*}}^{2,0}(\hat{s})+\sum_{q} \int_{\tau_{0}}^{1} \mathrm{~d} \tau \frac{\mathrm{d} L_{q \bar{q}}}{\mathrm{~d} \tau} \mathrm{d} \hat{\sigma}_{q \bar{q} \rightarrow \tilde{b}_{\alpha} \tilde{b}_{\alpha}^{*}}^{2,0}(\hat{s}) \\
& +\int_{\tau_{0}}^{1} \mathrm{~d} \tau \frac{\mathrm{d} L_{b \bar{b}}}{\mathrm{~d} \tau} \mathrm{d} \hat{\sigma}_{b \bar{b} \rightarrow \tilde{b}_{\alpha} \tilde{b}_{\alpha}^{*}}^{2,0}(\hat{s})
\end{aligned}
$$

where $\tau_{0}=4 m_{\tilde{b}_{\alpha}}^{2} / S$ is the production threshold. $S$ and $\hat{s}=\tau S$ are the squared center-ofmass (c.m.) energies of the hadronic and partonic processes, respectively. The sum runs over $q=u, d, c, s$. The three classes of partonic processes contributing are

$$
\begin{aligned}
g\left(p_{1}\right) g\left(p_{2}\right) & \rightarrow \tilde{b}_{\alpha}\left(p_{3}\right) \tilde{b}_{\alpha}^{*}\left(p_{4}\right), \\
q\left(p_{1}\right) \bar{q}\left(p_{2}\right) & \rightarrow \tilde{b}_{\alpha}\left(p_{3}\right) \tilde{b}_{\alpha}^{*}\left(p_{4}\right), \\
b\left(p_{1}\right) \bar{b}\left(p_{2}\right) & \rightarrow \tilde{b}_{\alpha}\left(p_{3}\right) \tilde{b}_{\alpha}^{*}\left(p_{4}\right) .
\end{aligned}
$$

The corresponding partonic cross section can be obtained from the Feynman diagrams in figure 10. In terms of the Mandelstam variables,

$$
\hat{s}=\left(p_{1}+p_{2}\right)^{2}, \quad \hat{t}=\left(p_{1}-p_{3}\right)^{2}, \quad \hat{u}=\left(p_{1}-p_{4}\right)^{2},
$$

the differential partonic cross section for a given subprocess $\xi \xi^{\prime} \rightarrow \tilde{b}_{\alpha} \tilde{b}_{\alpha}^{*}$ can be written as

$$
\mathrm{d} \hat{\sigma}_{\xi \xi^{\prime} \rightarrow \tilde{b}_{\alpha} \tilde{b}_{\alpha}^{*}}^{2,0}(\hat{s})=\bar{\sum}\left|\mathcal{M}_{\xi \xi^{\prime} \rightarrow \tilde{b}_{\alpha} \tilde{b}_{\alpha}^{*}}^{1,0}\right|^{2} \frac{\mathrm{d} \hat{t}}{16 \pi \hat{s}^{2}},
$$

with the squared lowest order matrix element averaged (summed) over initial (final) state spin and color. 


\subsection{Tree-level EW contributions}

The tree-level electroweak (EW) contributions, which are of the order $\mathcal{O}\left(\alpha_{s} \alpha\right)$ and $\mathcal{O}\left(\alpha^{2}\right)$, read as follows,

$$
\begin{aligned}
\mathrm{d} \sigma_{P P \rightarrow \tilde{b}_{\alpha} \tilde{b}_{\alpha}^{*}}^{\mathrm{LOEW}}(S)= & \int_{\tau_{0}}^{1} \mathrm{~d} \tau \frac{\mathrm{d} L_{g \gamma}}{\mathrm{d} \tau} \mathrm{d} \hat{\sigma}_{g \gamma \rightarrow \tilde{b}_{\alpha} \tilde{b}_{\alpha}^{*}}^{1,1}(\hat{s})+\sum_{q} \int_{\tau_{0}}^{1} \mathrm{~d} \tau \frac{\mathrm{d} L_{q \bar{q}}}{\mathrm{~d} \tau} \mathrm{d} \hat{\sigma}_{q \bar{q} \rightarrow \tilde{b}_{\alpha} \tilde{b}_{\alpha}^{*}}^{0,2}(\hat{s}) \\
& +\int_{\tau_{0}}^{1} \mathrm{~d} \tau \frac{\mathrm{d} L_{b \bar{b}}}{\mathrm{~d} \tau}\left[\mathrm{d} \hat{\sigma}_{b \bar{b} \rightarrow \tilde{b}_{\alpha} \tilde{b}_{\alpha}^{*}}^{1,1}(\hat{s})+\mathrm{d} \hat{\sigma}_{b \bar{b} \rightarrow \tilde{b}_{\alpha} \tilde{b}_{\alpha}^{*}}^{0,2}(\hat{s})\right] .
\end{aligned}
$$

The contributions of $\mathcal{O}\left(\alpha^{2}\right)$ arise from the processes $(2.3 \mathrm{~b})$ and (2.3c). The partonic cross sections,

$$
\mathrm{d} \hat{\sigma}_{q \bar{q} \rightarrow \tilde{b}_{\alpha} \tilde{b}_{\alpha}^{*}}^{0,2}(\hat{s})=\bar{\sum}\left|\mathcal{M}_{q \bar{q} \rightarrow \tilde{b}_{\alpha} \tilde{b}_{\alpha}^{*}}^{0,1}\right|^{2} \frac{\mathrm{d} \hat{t}}{16 \pi \hat{s}^{2}}, \quad \mathrm{~d} \hat{\sigma}_{b \bar{b} \rightarrow \tilde{b}_{a} \tilde{b}_{a}^{*}}^{0,2}(\hat{s})=\bar{\sum}\left|\mathcal{M}_{b \bar{b} \rightarrow \tilde{b}_{\alpha} \tilde{b}_{\alpha}^{*}}^{0,1}\right|^{2} \frac{\mathrm{d} \hat{t}}{16 \pi \hat{s}^{2}},
$$

are obtained from the diagrams in figure 10. In the case of process $(2.3 \mathrm{c})$, the diagrams with $t$-channel gluino and neutralino exchange further allow for a non-vanishing QCD-EW interference term of $\mathcal{O}\left(\alpha_{s} \alpha\right)$,

$$
\mathrm{d} \hat{\sigma}_{b \bar{b} \rightarrow \tilde{b}_{\alpha} \tilde{b}_{\alpha}^{*}}^{1,1}(\hat{s})=2 \bar{\sum} \operatorname{Re}\left\{\mathcal{M}_{b \bar{b} \rightarrow \tilde{b}_{\alpha} \tilde{b}_{\alpha}^{*}}^{1,0}\left(\mathcal{M}_{b \bar{b} \rightarrow \tilde{b}_{\alpha} \tilde{b}_{\alpha}^{*}}^{0,1}\right)^{*}\right\} \frac{\mathrm{d} \hat{t}}{16 \pi \hat{s}^{2}} .
$$

We approximate the CKM matrix $V$ with the unity matrix, thus we consistently neglect the diagram depicted in figure 10(d). The latter diagram, together with the diagrams in figure $10(\mathrm{~b})$, gives rise to $\mathcal{O}\left(\alpha_{s} \alpha+\alpha^{2}\right)$ contributions. These contributions are at least quadratic in $\left|V_{c b}\right| \approx 10 \cdot\left|V_{u b}\right| \approx 4 \cdot 10^{-2}$ and numerically negligible (see section 4.3).

The first $\mathcal{O}\left(\alpha_{s} \alpha\right)$ contribution in eq. (2.6) arises from the photon-gluon induced process

$$
g\left(p_{1}\right) \gamma\left(p_{2}\right) \rightarrow \tilde{b}_{\alpha}\left(p_{3}\right) \tilde{b}_{\alpha}^{*}\left(p_{4}\right) .
$$

The corresponding partonic cross section can be obtained from the diagrams in figure 11 and reads as follows

$$
\mathrm{d} \hat{\sigma}_{g \gamma \rightarrow \tilde{b}_{\alpha} \tilde{b}_{\alpha}^{*}}^{1,1}(\hat{s})=\bar{\sum}\left|\mathcal{M}_{g \gamma \rightarrow \tilde{b}_{\alpha} \tilde{b}_{\alpha}^{*}}^{1 / 2,1 / 2}\right|^{2} \frac{\mathrm{d} \hat{t}}{16 \pi \hat{s}^{2}} .
$$

\section{Next-to-leading order EW contributions}

In this section we list the NLO EW corrections to the process (1.2). These contributions are of the order $\mathcal{O}\left(\alpha_{s}^{2} \alpha\right)$ and arise from virtual corrections and bremsstrahlung processes. Using an obvious notation, the corresponding contributions to the total cross section read as follows

$$
\begin{aligned}
\mathrm{d} \sigma_{P P \rightarrow \tilde{b}_{\alpha} \tilde{b}_{\alpha}^{*}}^{\mathrm{NLO} \mathrm{EW}}(S)= & \int_{\tau_{0}}^{1} \mathrm{~d} \tau \frac{\mathrm{d} L_{g g}}{\mathrm{~d} \tau}\left[\mathrm{d} \hat{\sigma}_{g g \rightarrow \tilde{b}_{\alpha} \tilde{b}_{\alpha}^{*}}^{2,1}(\hat{s})+\mathrm{d} \hat{\sigma}_{g g \rightarrow \tilde{b}_{\alpha} \tilde{b}_{\alpha}^{*} \gamma}^{2,1}(\hat{s})\right] \\
& +\sum_{q} \int_{\tau_{0}}^{1} \mathrm{~d} \tau \frac{\mathrm{d} L_{q \bar{q}}}{\mathrm{~d} \tau}\left[\mathrm{d} \hat{\sigma}_{q \bar{q} \rightarrow \tilde{b}_{\alpha} \tilde{b}_{\alpha}^{*}}^{2,1}(\hat{s})+\mathrm{d} \hat{\sigma}_{q \bar{q} \rightarrow \tilde{b}_{\alpha} \tilde{b}_{\alpha}^{*} \gamma}^{2,1}(\hat{s})+\mathrm{d} \hat{\sigma}_{q \bar{q} \rightarrow \tilde{b}_{\alpha} \tilde{b}_{\alpha}^{*} g}^{2,1}(\hat{s})\right] \\
& +\sum_{q} \int_{\tau_{0}}^{1} \mathrm{~d} \tau\left[\frac{\mathrm{d} L_{q g}}{\mathrm{~d} \tau} \mathrm{d} \hat{\sigma}_{g q \rightarrow \tilde{b}_{\alpha} \tilde{b}_{\alpha}^{*} q}^{2,1}(\hat{s})+\frac{\mathrm{d} L_{\bar{q} g}}{\mathrm{~d} \tau} \mathrm{d} \hat{\sigma}_{g \bar{q}^{2} \rightarrow \tilde{b}_{\alpha} \tilde{b}_{\alpha}^{*} \bar{q}}^{2,1}(\hat{s})\right]
\end{aligned}
$$


We do not consider the contributions arising from the bremsstrahlung processes

$$
\gamma\left(p_{1}\right) q\left(p_{2}\right) \rightarrow \tilde{b}_{\alpha}\left(p_{3}\right) \tilde{b}_{\alpha}^{*}\left(p_{4}\right) q\left(p_{5}\right), \quad \gamma\left(p_{1}\right) \bar{q}\left(p_{2}\right) \rightarrow \tilde{b}_{\alpha}\left(p_{3}\right) \tilde{b}_{\alpha}^{*}\left(p_{4}\right) \bar{q}\left(p_{5}\right) .
$$

As already pointed out in ref. [32], they are suppressed because of the $\mathcal{O}(\alpha)$ suppression of the photon PDF inside the proton. Moreover, these processes are further suppressed by an additional factor $\alpha_{s}$ with respect to the process (2.9) and thus negligible. The $\mathcal{O}\left(\alpha_{s}^{2} \alpha\right)$ contributions of the partonic processes with a bottom quark in the initial state are neglected as well. The reason is twofold. First of all these contributions are suppressed by the bottom PDF with respect to the contributions in eq. (3.1). In addition they have an additional factor $\alpha_{s}$ with respect to the $\mathcal{O}\left(\alpha_{s} \alpha+\alpha^{2}\right)$ contributions of the process (2.3c), which turn out to be small (cf. section 4.3).

The amplitudes are generated and algebraically simplified with support of FeynArts [40, 41] and FormCalc [41, 42], while the numerical evaluation of the one-loop integrals has been performed using LoopTools [42]. Infrared (IR) singularities are regularized giving a small mass $\lambda_{\gamma}$ and $\lambda_{g}$ to the photon and to the gluon, respectively. The mass of the light quarks is kept in order to regularize the collinear singularities.

\subsection{Virtual corrections}

The $\mathcal{O}\left(\alpha_{s}^{2} \alpha\right)$ contributions to the partonic process (2.3a) are given by

$$
\mathrm{d} \hat{\sigma}_{g g \rightarrow \tilde{b}_{\alpha} \tilde{b}_{\alpha}^{*}}^{2,1}(\hat{s})=2 \bar{\sum} \operatorname{Re}\left\{\mathcal{M}_{g g \rightarrow \tilde{b}_{\alpha} \tilde{b}_{\alpha}^{*}}^{1,0}\left(\mathcal{M}_{g g \rightarrow \tilde{b}_{\alpha} \tilde{b}_{\alpha}^{*}}^{1,1}\right)^{*}\right\} \frac{\mathrm{d} \hat{t}}{16 \pi \hat{s}^{2}},
$$

where $\mathcal{M}^{1,0}$ is the tree-level amplitude while $\mathcal{M}^{1,1}$ is the one-loop amplitude obtained from the diagrams depicted in figure 12 . The virtual corrections to the process $(2.3 \mathrm{~b})$ read as follows

$$
\mathrm{d} \hat{\sigma}_{q \bar{q} \rightarrow \tilde{b}_{\alpha} \tilde{b}_{\alpha}^{*}}^{2,1}(\hat{s})=2 \bar{\sum} \operatorname{Re}\left\{\mathcal{M}_{q \bar{q} \rightarrow \tilde{b}_{\alpha} \tilde{b}_{\alpha}^{*}}^{1,0}\left(\mathcal{M}_{q \bar{q} \rightarrow \tilde{b}_{\alpha} \tilde{b}_{\alpha}^{*}}^{1,1}\right)^{*}+\mathcal{M}_{q \bar{q} \rightarrow \tilde{b}_{\alpha} \tilde{b}_{\alpha}^{*}}^{0,1}\left(\mathcal{M}_{q \bar{q} \rightarrow \tilde{b}_{\alpha} \tilde{b}_{\alpha}^{*}}^{2,0}\right)^{*}\right\} \frac{\mathrm{d} \hat{t}}{16 \pi \hat{s}^{2}} .
$$

$\mathcal{M}^{0,1}$ and $\mathcal{M}^{1,0}$ are the tree-level EW and the tree-level QCD amplitudes, respectively. $\mathcal{M}^{1,1}$ is the one-loop amplitude obtained from the EW insertions to the leading-order diagrams and from the QCD corrections to the tree-level EW diagrams (figure 13). $\mathcal{M}^{2,0}$ is the amplitude corresponding to the QCD box diagrams depicted in figure 14 .

In order to cancel the UV divergences we need the $\mathcal{O}(\alpha)$ renormalization of the wavefunction of the light quarks and of the sbottom sector. The field renormalization constants are fixed in the on-shell scheme, in analogy to that described in ref. [32]. The renormalization of the sbottom sector has to be performed together with that of the stop sector. In order to avoid numerical instabilities and artificially big contributions from the counterterms, care has to be taken in choosing the renormalization scheme [37, 38]. We use the "DR bottom-quark mass" scheme introduced in ref. [37]. Since in particular regions of the MSSM parameter space this scheme can give rise to numerical instabilities, we have explicitly checked its reliability in the SUSY scenarios considered in this paper (cf. section 4). The explicit expression of the renormalization constants in the "DR bottom-quark mass" scheme are collected in appendix A. 


\subsection{Real corrections}

The $\mathcal{O}\left(\alpha_{s}^{2} \alpha\right)$ contributions to the partonic processes with a photon in the final state,

$$
\begin{aligned}
g\left(p_{1}\right) g\left(p_{2}\right) & \rightarrow \tilde{b}_{\alpha}\left(p_{3}\right) \tilde{b}_{\alpha}^{*}\left(p_{4}\right) \gamma\left(p_{5}\right), \\
q\left(p_{1}\right) \bar{q}\left(p_{2}\right) & \rightarrow \tilde{b}_{\alpha}\left(p_{3}\right) \tilde{b}_{\alpha}^{*}\left(p_{4}\right) \gamma\left(p_{5}\right),
\end{aligned}
$$

are obtained from the tree-level diagrams in figure 15. The phase-space integration is divergent in the soft photon region, i.e. in the region $p_{5}^{0} \rightarrow 0$. In the case of the process $(3.5 \mathrm{~b})$ further singularities arise in the collinear region, i.e. $p_{1,2} \cdot p_{5} \rightarrow 0$. IR and collinear singularities are treated using the phase-space slicing method. The description of the method and the relevant formulae are collected in ref. [28].

The gluon bremsstrahlung process,

$$
q\left(p_{1}\right) \bar{q}\left(p_{2}\right) \rightarrow \tilde{b}_{\alpha}\left(p_{3}\right) \tilde{b}_{\alpha}^{*}\left(p_{4}\right) g\left(p_{5}\right),
$$

contributes at $\mathcal{O}\left(\alpha_{s}^{2} \alpha\right)$ via the interference of QCD-based and EW-based Feynman diagrams depicted in figure 16. IR singularities of gluonic origin are treated in close analogy to the photonic case. Color correlations are taken into account using the formulae collected in appendix B of ref. [28]. Due to the color structure, the interference term of a QCD-based and an EW-based diagram vanishes if both gluons are emitted from an initial-state or a final-state particle.

Real quark radiation contributes at $\mathcal{O}\left(\alpha_{s}^{2} \alpha\right)$ as well,

$$
\begin{aligned}
& g\left(p_{1}\right) q\left(p_{2}\right) \rightarrow \tilde{b}_{\alpha}\left(p_{3}\right) \tilde{b}_{\alpha}^{*}\left(p_{4}\right) q\left(p_{5}\right), \\
& g\left(p_{1}\right) \bar{q}\left(p_{2}\right) \rightarrow \tilde{b}_{\alpha}\left(p_{3}\right) \tilde{b}_{\alpha}^{*}\left(p_{4}\right) \bar{q}\left(p_{5}\right) .
\end{aligned}
$$

This IR- and collinear-finite set is given by the interference of QCD and EW tree-level diagrams (cf. figure 17). Only the interference from initial-state and final-state radiation contributes.

The IR singularities arising in the $g g$ channel cancel in the sum of virtual corrections, process (3.3), and real photon radiation (3.5a). In the $q \bar{q}$ channel the sum of the virtual corrections (3.4) and of the contributions of real photon radiation (3.5b) and real gluon radiation (3.6) is IR finite. This sum is affected by universal collinear singularities of photonic origin that can be absorbed in the PDFs. This can be achieved by means of the following substitution [43],

$$
f_{q}\left(x, \mu_{F}\right) \rightarrow f_{q}\left(x, \mu_{F}\right)\left(1-\frac{\alpha e_{q}^{2}}{\pi} \kappa_{v+s}\right)-\frac{\alpha e_{q}^{2}}{2 \pi} \int_{x}^{1-\delta_{s}} \frac{\mathrm{d} z}{z} f_{q}\left(\frac{x}{z}, \mu_{F}\right) \kappa_{c}(z) .
$$

$e_{q}$ is the electric charge of quark $q$ expressed in units of the positron charge, while

$$
\begin{aligned}
& \kappa_{v+s}=1-\ln \delta_{s}-\ln ^{2} \delta_{s}+\left(\ln \delta_{s}+\frac{3}{4}\right) \ln \left(\frac{\mu_{F}^{2}}{m_{q}^{2}}\right)+\frac{1}{4}\left(9+\frac{2 \pi^{2}}{3}+3 \ln \delta_{s}-2 \ln ^{2} \delta_{s}\right), \\
& \kappa_{c}(z)=P_{q q}(z) \ln \left(\frac{\mu_{F}^{2}}{m_{q}^{2}} \frac{1}{(1-z)^{2}}-1\right)-\left[P_{q q}(z) \ln \left(\frac{1-z}{z}\right)-\frac{3}{2} \frac{1}{1-z}+2 z+3\right],
\end{aligned}
$$


with the splitting function $P_{q q}(z)=\left(1+z^{2}\right) /(1-z)$. The factorization is performed in the DIS scheme. The replacement of the PDFs in eq. (2.1) gives further contributions of $\mathcal{O}\left(\alpha_{s}^{2} \alpha\right)$ to the total cross section. As already mentioned, they cancel the collinear singularities affecting the $\mathcal{O}\left(\alpha_{s}^{2} \alpha\right)$ contributions (3.1).

\subsection{Resummation in the $\mathrm{b} / \tilde{\mathrm{b}}$ sector}

The Higgs sector in the MSSM corresponds to a type-II two-Higgs doublet model, i.e. the down-type quarks couple to $H_{1}$ and the up-type quarks to $H_{2}$. After spontaneous symmetry breaking, the up- (down-)type quarks get their mass by their coupling to the vacuum expectation value $v_{2}\left(v_{1}\right)$ of $H_{2}\left(H_{1}\right)$. At tree level, the bottom-quark mass $m_{b}$ is related to the $H_{1} b \bar{b}$ Yukawa coupling $\lambda_{b}$ via

$$
m_{b}=\lambda_{b} v_{1}
$$

Radiative corrections induces and effective $\mathrm{H}_{2} b \bar{b}$ coupling that can significantly alter the tree-level relation (3.10) [39, 44-47]. This higher-order contributions do not decouple at low energies and are enhanced by a factor $\tan \beta=v_{2} / v_{1}$. As shown in ref. [39], the leading $\tan \beta$ enhanced terms can be resummed by using an appropriate effective bottom-quark Yukawa coupling. We follow ref. [37] and use an effective Yukawa coupling defined as follows,

$$
\bar{\lambda}_{b}=\frac{1}{v_{1}} \frac{m_{b}^{\overline{\mathrm{DR}}}\left(\mu_{R}\right)+m_{b} \Delta m_{b}}{1+\Delta m_{b}} \equiv \frac{m_{b}^{\overline{\mathrm{DR}}, \mathrm{eff}}}{v_{1}}
$$

where $\Delta m_{b}$ is given by

$$
\begin{aligned}
\Delta m_{b}= & \frac{2 \alpha_{s}}{3 \pi} M_{\tilde{g}} \mu \tan \beta I\left(m_{\tilde{b}_{1}}, m_{\tilde{b}_{2}}, m_{\tilde{g}}\right)+\frac{\lambda_{t}^{2}}{16 \pi^{2}} \mu A_{t} \tan \beta I\left(m_{\tilde{t}_{1}}, m_{\tilde{t}_{2}}, \mu\right) \\
& -\frac{g^{2}}{16 \pi^{2}} \mu M_{2} \tan \beta\left[\cos ^{2} \theta_{\tilde{t}} I\left(m_{\tilde{t}_{1}}, M_{2}, \mu\right)+\sin ^{2} \theta_{\tilde{t}_{2}} I\left(m_{\tilde{t}_{2}}, M_{2}, \mu\right)\right. \\
& \left.+\frac{1}{2} \cos ^{2} \theta_{\tilde{b}} I\left(m_{\tilde{b}_{1}}, M_{2}, \mu\right)+\frac{1}{2} \sin ^{2} \theta_{\tilde{b}_{2}} I\left(m_{\tilde{b}_{2}}, M_{2}, \mu\right)\right], \\
I(a, b, c)= & \frac{1}{\left(a^{2}-b^{2}\right)\left(b^{2}-c^{2}\right)\left(a^{2}-c^{2}\right)}\left[a^{2} b^{2} \log \frac{a^{2}}{b^{2}}+b^{2} c^{2} \log \frac{b^{2}}{c^{2}}+c^{2} a^{2} \log \frac{c^{2}}{a^{2}}\right] .
\end{aligned}
$$

Large logarithms from the running of the Yukawa coupling $\lambda_{b}$ at the renormalization scale $\mu_{R}$ are resummed using the $\overline{\mathrm{DR}}$ bottom-quark mass,

$$
m_{b}^{\overline{\mathrm{DR}}}\left(\mu_{R}\right)=m_{b}^{\mathrm{OS}}+\frac{m_{b}}{2}\left(\Sigma_{b L}^{\mathrm{fin}} \cdot\left(m_{b}\right)+\Sigma_{b R}^{\mathrm{fin}}\left(m_{b}\right)+2 \Sigma_{b S}^{\mathrm{fin}} \cdot\left(m_{b}\right)\right) .
$$

$m_{b}^{\mathrm{OS}}$ is the on-shell bottom-quark mass defined according to

$$
m_{b}^{\mathrm{OS}}=m_{b}^{\overline{\mathrm{MS}}}\left(m_{Z}\right) b^{\text {shift }}, \quad b^{\text {shift }}=1+\frac{\alpha_{s}}{\pi}\left(\frac{4}{3}-\log \frac{\left(m_{b}^{\overline{\mathrm{MS}}}\right)^{2}}{m_{Z}^{2}}\right) .
$$

$\Sigma^{\text {fin. }}$ is the finite part of the scalar self-energies defined according to the Lorentz decomposition (A.7) of the appendix A. The term proportional to $\Delta m_{b}$ in the numerator 
of eq. (3.11) has to be inserted to avoid double counting of the one-loop contributions of the resummed terms.

Further $\tan \beta$ enhancement effects arise from three-point functions involving Higgsbottom vertices. The $\tan \beta$-enhanced terms can be taken into account by modifying the $\mathcal{H} b \bar{b}$ coupling $g_{\mathcal{H} b b \bar{b}}{ }^{1}$ The combined effect of the resummation in the relation between $\lambda_{b}$ and $m_{b}$ and of the resummation in the Higgs-bottom vertices is accounted for by performing the following substitutions,

$$
\begin{array}{ll}
\left.g_{h^{0} b b} \rightarrow g_{h^{0} b b}\right|_{\lambda_{b} \rightarrow \bar{\lambda}_{b}}\left(1-\frac{\Delta m_{b}}{\tan \beta \tan \alpha}\right), & \left.g_{A^{0} b b} \rightarrow g_{A^{0} b b}\right|_{\lambda_{b} \rightarrow \bar{\lambda}_{b}}\left(1-\frac{\Delta m_{b}}{\tan \beta^{2}}\right), \\
\left.g_{H^{0} b b} \rightarrow g_{H^{0} b b}\right|_{\lambda_{b} \rightarrow \bar{\lambda}_{b}}\left(1+\Delta m_{b} \frac{\tan \alpha}{\tan \beta}\right), & g_{G^{0} b b} \rightarrow g_{G^{0} b b} .
\end{array}
$$

The coupling involving the Goldstone boson $G^{0}$ is not modified since the contribution from the vertex corrections exactly compensates the contribution of the bottom-Yukawa coupling resummation.

\section{Numerical results}

In this section we perform a detailed numerical analysis for diagonal sbottom-pair production at NLO EW. We stick to the notation introduced in ref. [28]. The leading order cross section, the tree-level EW and the NLO EW contributions to the cross section are labeled by

$$
\sigma^{\text {Born }}=\sigma^{2,0}, \quad \Delta \sigma^{\text {tree } \mathrm{EW}}=\left(\sigma^{1,1}+\sigma^{0,2}\right), \quad \Delta \sigma^{\mathrm{NLO} \mathrm{EW}}=\sigma^{2,1},
$$

respectively. $\Delta \sigma^{\mathrm{EW}}=\Delta \sigma^{\text {tree } \mathrm{EW}}+\Delta \sigma^{\mathrm{NLO}} \mathrm{EW}$ will be referred to as the $\mathrm{EW}$ contribution. The total sum of the LO cross section with the EW contributions is denoted by $\sigma^{\mathrm{NLO}}=$ $\sigma^{\text {Born }}+\Delta \sigma^{\mathrm{EW}}$. Relative EW contributions are defined by

$$
\delta^{\text {tree } \mathrm{EW}}=\Delta \sigma^{\text {tree } \mathrm{EW}} / \sigma^{\mathrm{Born}}, \quad \delta^{\mathrm{NLO} \mathrm{EW}}=\Delta \sigma^{\mathrm{NLO} \mathrm{EW}} / \sigma^{\mathrm{Born}}, \quad \delta^{\mathrm{EW}}=\Delta \sigma^{\mathrm{EW}} / \sigma^{\text {Born }} .
$$

In distributions $\delta$ denotes the relative $\mathrm{EW}$ contribution defined as $\delta=\left(\mathcal{O}_{\mathrm{NLO}}-\right.$ $\left.\mathcal{O}_{\text {Born }}\right) / \mathcal{O}_{\text {Born }}$, where $\mathcal{O}$ is a generic observable and $\mathcal{O}_{\mathrm{NLO}}$ is the sum of the Born and the EW contributions.

\subsection{Input parameters}

The Standard Model input parameters are chosen in correspondence with [48, 49],

$$
\begin{aligned}
M_{Z} & =91.1876 \mathrm{GeV}, & M_{W} & =80.399 \mathrm{GeV}, \\
\alpha^{-1} & =137.036, & \alpha_{s}\left(M_{Z}\right) & =0.119, \\
m_{t} & =170.9 \mathrm{GeV}, & m_{b}^{\overline{\mathrm{MS}}}\left(m_{Z}\right) & =2.94 \mathrm{GeV} .
\end{aligned}
$$

The strong coupling constant $\alpha_{s}$ has been defined in the $\overline{\mathrm{MS}}$ scheme using the two-loop renormalization group equation with five active flavors.

\footnotetext{
${ }^{1} \mathcal{H}$ stands for any of the neutral Higgs and Goldstone bosons, i.e. $\mathcal{H}=h^{0}, H^{0}, A^{0}, G^{0}$.
} 


\begin{tabular}{|cccccc|}
\hline & $\boldsymbol{m}_{\mathbf{0}}$ & $\boldsymbol{m}_{\mathbf{1} \boldsymbol{2}}$ & $\boldsymbol{A}_{\mathbf{0}}$ & $\tan \boldsymbol{\beta}$ & $\operatorname{sign}(\boldsymbol{\mu})$ \\
SPS1a $^{\prime}$ & $70 \mathrm{GeV}$ & $250 \mathrm{GeV}$ & $-300 \mathrm{GeV}$ & 10 & + \\
SPS4 & $400 \mathrm{GeV}$ & $300 \mathrm{GeV}$ & 0 & 49.4 & + \\
\hline & $\boldsymbol{\Lambda}$ & $\boldsymbol{M}_{\text {mess }}$ & $\boldsymbol{N}_{\text {mess }}$ & $\tan \boldsymbol{\beta}$ & $\operatorname{sign}(\boldsymbol{\mu})$ \\
SPS8 & $100 \mathrm{TeV}$ & $200 \mathrm{TeV}$ & 1 & 15 & + \\
\hline
\end{tabular}

Table 1. High-energy input parameters for the different SUSY scenarios considered. The mass parameters $m_{0}, m_{1 / 2}$ and $A_{0}$ are given at the GUT scale, $\tan \beta$ is evaluated at $M_{\mathrm{SUSY}}=1 \mathrm{TeV}$.

\begin{tabular}{|cccccccc|}
\hline & $\boldsymbol{\Delta}_{\boldsymbol{m}}$ & $\boldsymbol{m}_{\boldsymbol{b}}^{\overline{\mathrm{DR}}, \mathrm{eff}}$ & $\tilde{\boldsymbol{b}}_{\mathbf{1}}$ & $\tilde{\boldsymbol{b}}_{\boldsymbol{2}}$ & $\tilde{\boldsymbol{g}}$ & $\tilde{\boldsymbol{\chi}}_{\mathbf{1}}^{0}$ & $\tilde{\boldsymbol{\chi}}_{\mathbf{1}}^{ \pm}$ \\
\hline SPS1a $^{\prime}$ & 0.037 & 2.38 & 500 & 533 & 609 & 101 & 180 \\
SPS4 & 0.23 & 2.05 & 428 & 633 & 736 & 123 & 217 \\
SPS8 & 0.03 & 2.42 & 1070 & 1085 & 141 & 253 & \\
\hline
\end{tabular}

Table 2. The shift $\Delta m_{b}$ and the resulting effective bottom-quark mass as well as the on-shell masses of the bottom squarks, of the gluino, and of the lightest neutralino and chargino within the different SUSY scenarios considered. $\Delta m_{b}$ and $m_{b}^{\overline{\mathrm{DR}} \text {,eff }}$ are evaluated at the scale used for $\tilde{b}_{1} \tilde{b}_{1}^{*}$ production. All masses are given in $\mathrm{GeV}$.

For the numerical analysis we consider the mSUGRA scenarios SPS1a' and SPS4. The first one is a "typical" SUSY scenario proposed by the SPA convention for comparison with other calculations [50]. The scenario SPS4 is characterized by a large value of $\tan \beta$. Within this scenario we study the dependence of the total cross section on the squark masses and on $\tan \beta$. The third scenario considered is the GMSB scenario SPS8.

The particle spectrum is determined following the procedure described in ref. [28]. Starting from GUT-scale parameters, cf. table 1, we use the program Softsusy [51] to evolve the soft-breaking parameters down to the SUSY scale $M_{\text {SUSY }}$. In accordance to the SPA convention, a common SUSY scale $M_{\mathrm{SUSY}}=1 \mathrm{TeV}$ has been chosen. To get the right mixing in the sbottom sector, we first compute the low energy masses and use these to calculate the effective bottom-quark mass $m_{b}^{\overline{\mathrm{DR}}}$,eff , eq. (3.11). This mass is then used in the bottom-squark mass matrix to calculate the sbottom-mass eigenstates. The lighter of the two bottom-squarks is taken as the dependent squark. Its mass is therefore fixed by $\mathrm{SU}(2)$ invariance. Table 2 collects the shift $\Delta m_{b}$, cf. eq. (3.12), and the effective bottomquark mass together with the on-shell mass of the bottom squarks, of the gluino and of the lightest neutralino and chargino.

Unless otherwise stated, the results presented in this section are computed setting the hadronic center of mass energy to $\sqrt{S}=14 \mathrm{TeV}$ and using the MRST2004QED parton distribution functions [52]. The factorization and renormalization scales are set to a common value, $\mu=\mu_{R}=\mu_{F}=m_{\tilde{b}_{\alpha}}$, i.e. to the mass of the produced bottom squark.

\subsection{Total hadronic cross section}

Table 3 shows the hadronic cross section for diagonal bottom-squark production within the three considered scenarios for $\sqrt{S}=14 \mathrm{TeV}$. 


\begin{tabular}{|c|c|c|c|c|c|}
\hline $14 \mathrm{TeV}$ & $\begin{array}{l}\boldsymbol{\sigma}^{\text {Born }} \\
\mathcal{O}\left(\alpha_{s}^{2}\right)\end{array}$ & $\begin{array}{c}\Delta \boldsymbol{\sigma}^{\text {tree } \mathbf{E W}} \\
\mathcal{O}\left(\alpha_{s} \alpha+\alpha^{2}\right)\end{array}$ & $\begin{array}{c}\Delta \sigma^{\mathrm{g} \gamma} \\
\mathcal{O}\left(\alpha_{s} \alpha\right)\end{array}$ & $\begin{array}{c}\Delta \boldsymbol{\sigma}^{\text {NLO EW }} \\
\mathcal{O}\left(\alpha_{s}^{2} \alpha\right)\end{array}$ & $\begin{array}{c}\boldsymbol{\Delta} \boldsymbol{\sigma}^{\mathbf{E W}} \\
\mathcal{O}\left(\alpha_{s} \alpha+\alpha^{2}+\alpha_{s}^{2} \alpha\right)\end{array}$ \\
\hline \multicolumn{6}{|l|}{ SPS1a $^{\prime}$} \\
\hline \multirow[t]{2}{*}{$\tilde{b}_{1} \tilde{b}_{1}^{*}$} & $444.3(3)$ & 0.8 & 2.0 & -6.0 & -3.2 \\
\hline & & $0.2 \%$ & $0.5 \%$ & $-1.4 \%$ & $-0.7 \%$ \\
\hline \multirow[t]{2}{*}{$\tilde{b}_{2} \tilde{b}_{2}^{*}$} & $310.3(1)$ & $\ll 0.1$ & 1.5 & -2.9 & -1.4 \\
\hline & & $\approx 0 \%$ & $0.5 \%$ & $-0.9 \%$ & $-0.5 \%$ \\
\hline \multicolumn{6}{|l|}{ SPS4 } \\
\hline \multirow[t]{2}{*}{$\tilde{b}_{1} \tilde{b}_{1}^{*}$} & $1050.9(3)$ & -0.4 & 4.3 & -19.4 & -15.5 \\
\hline & & $\approx 0 \%$ & $0.4 \%$ & $-1.8 \%$ & $-1.5 \%$ \\
\hline \multirow[t]{2}{*}{$\tilde{b}_{2} \tilde{b}_{2}^{*}$} & $112.36(6)$ & 0.27 & 0.61 & -2.85 & -1.97 \\
\hline & & $0.2 \%$ & $0.5 \%$ & $-2.5 \%$ & $-1.8 \%$ \\
\hline \multicolumn{6}{|l|}{ SPS8 } \\
\hline \multirow[t]{2}{*}{$\tilde{b}_{1} \tilde{b}_{1}^{*}$} & $3.405(1)$ & 0.002 & 0.029 & -0.003 & 0.028 \\
\hline & & $0.1 \%$ & $0.9 \%$ & $-0.1 \%$ & $0.8 \%$ \\
\hline \multirow[t]{2}{*}{$\tilde{b}_{2} \tilde{b}_{2}^{*}$} & $3.042(1)$ & 0.007 & 0.026 & 0.008 & 0.042 \\
\hline & & $0.2 \%$ & $0.9 \%$ & $0.3 \%$ & $1.4 \%$ \\
\hline
\end{tabular}

Table 3. Hadronic cross section for diagonal $\tilde{b}_{\alpha} \tilde{b}_{\alpha}^{*}$ production at the $14 \mathrm{TeV}$ LHC within three different scenarios. Shown are the LO cross section, the tree-level EW as well as NLO EW contributions and the relative corrections as defined in the text. The numbers in brackets refer to the integration uncertainty in the last digit and are omitted if the uncertainty is at least one order of magnitude smaller than the quoted precision. All cross sections are given in femtobarn (fb).

As expected, the total cross section is dominated by the LO QCD contribution of $\mathcal{O}\left(\alpha_{s}^{2}\right)$. The contribution of the photon-induced process is independent of the mixing angle. In each scenario, its yield relative to the leading order cross section is similar for the two processes considered. Although formally suppressed by a factor $\alpha_{s}$, the NLO EW corrections are typically bigger than the tree-level EW contributions. In the SPS1a' (SPS4) scenario the tree level and NLO EW contributions are more important in case of $\tilde{b}_{1} \tilde{b}_{1}^{*}\left(\tilde{b}_{2} \tilde{b}_{2}^{*}\right)$ production. This can be explained by the chirality dependence of the $\mathrm{SU}(2)$ coupling and by the fact that in the SPS1a' (SPS4) scenario $\tilde{b}_{1}\left(\tilde{b}_{2}\right)$ is mostly left-handed.

In the SPS8 scenario the bottom squarks are twice as heavy as in the aforementioned scenarios, thus the Born cross section is about two orders of magnitude smaller. Further, the mixing between left- and right-handed squarks is more important and the sbottom masses are nearly degenerate. These features partially soften the differences among the tree-level EW contributions to $\tilde{b}_{1} \tilde{b}_{1}^{*}$ production and the ones to $\tilde{b}_{2} \tilde{b}_{2}^{*}$ production. ${ }^{2}$ Huge cancellations between the $q \bar{q}$ and the $g g$ channel amplify the dependence of the NLO EW contribution on the production process considered. As a result the NLO EW contributions

\footnotetext{
${ }^{2}$ In the no-mixing limit the tree-level EW contributions to $\tilde{b}_{L}$ production is one order of magnitude larger than the one contributing to $\tilde{b}_{R}$ production.
} 


\begin{tabular}{|cccccc|}
\hline \multirow{2}{*}{$\mathbf{7}$ TeV } & $\boldsymbol{\sigma}^{\text {Born }}$ & $\boldsymbol{\Delta} \boldsymbol{\sigma}^{\text {tree EW }}$ & $\boldsymbol{\Delta} \boldsymbol{\sigma}^{\mathbf{g} \boldsymbol{\gamma}}$ & $\boldsymbol{\Delta} \boldsymbol{\sigma}^{\text {NLO EW }}$ & $\boldsymbol{\Delta} \boldsymbol{\sigma}^{\text {EW }}$ \\
& $\mathcal{O}\left(\alpha_{s}^{2}\right)$ & $\mathcal{O}\left(\alpha_{s} \alpha+\alpha^{2}\right)$ & $\mathcal{O}\left(\alpha_{s} \alpha\right)$ & $\mathcal{O}\left(\alpha_{s}^{2} \alpha\right)$ & $\mathcal{O}\left(\alpha_{s} \alpha+\alpha^{2}+\alpha_{s}^{2} \alpha\right)$ \\
\hline SPS1a & & & & & \\
$\tilde{b}_{1} \tilde{b}_{1}^{*}$ & $30.42(2)$ & 0.10 & 0.20 & -0.35 & -0.05 \\
& & $0.3 \%$ & $0.7 \%$ & $-1.1 \%$ & $-0.2 \%$ \\
$\tilde{b}_{2} \tilde{b}_{2}^{*}$ & $19.286(6)$ & 0.004 & 0.136 & -0.203 & -0.064 \\
& & $\approx 0 \%$ & $0.7 \%$ & $-1.1 \%$ & $-0.3 \%$ \\
\hline SPS4 & & & & & \\
$\tilde{b}_{1} \tilde{b}_{1}^{*}$ & $89.10(2)$ & -0.01 & 0.52 & -1.69 & -1.18 \\
& & $\approx 0 \%$ & $0.6 \%$ & $-1.9 \%$ & $-1.3 \%$ \\
$\tilde{b}_{2} \tilde{b}_{2}^{*}$ & $5.175(2)$ & 0.023 & 0.043 & -0.125 & -0.059 \\
& & $0.5 \%$ & $0.8 \%$ & $-2.4 \%$ & $-1.1 \%$ \\
\hline SPS8 & & & & \\
$\tilde{b}_{1} \tilde{b}_{1}^{*}$ & $0.03706(1)$ & 0.00004 & 0.00059 & 0.00010 & 0.00073 \\
& & $0.1 \%$ & $1.6 \%$ & $0.3 \%$ & $2.0 \%$ \\
$\tilde{b}_{2} \tilde{b}_{2}^{*}$ & $0.03118(1)$ & 0.00011 & 0.00051 & 0.00030 & 0.00092 \\
& & $0.4 \%$ & $1.6 \%$ & $1.0 \%$ & $3.0 \%$ \\
\hline
\end{tabular}

Table 4 . Same as table 3 but considering diagonal $\tilde{b}_{\alpha} \tilde{b}_{\alpha}^{*}$ production at the $7 \mathrm{TeV}$ LHC.

to $b_{1} b_{1}^{*}$ production and the ones to $b_{2} b_{2}^{*}$ production have opposite sign, the latter being three times bigger than the former. Summing up the various contributions, the relative yield in the scenario considered is below $2 \%$.

Table 4 collects the hadronic cross section for $\sqrt{S}=7 \mathrm{TeV}$. The leading order total cross sections are reduced proportionally to the mass of the produced squark. They amount to $1-10 \%$ of their value at $\sqrt{S}=14 \mathrm{TeV}$. In all the scenarios considered, the contribution of the photon-induced channel is enhanced with respect to the $14 \mathrm{TeV}$ case. In the SPS1a' (SPS4) scenario the impact of the NLO EW contributions to the production of the mostly left-handed bottom-squark, $\tilde{b}_{1} \tilde{b}_{1}^{*}\left(\tilde{b}_{2} \tilde{b}_{2}^{*}\right)$, is reduced. In contrast, the NLO EW contributions become more important in case of the production of the mostly right-handed sbottom. In the SPS8 scenario the EW contributions of the various channels are enhanced. In particular the NLO EW contributions are positive for both production processes. This is a consequence of the enhancement of the NLO EW contributions to the $g g$ channel at $\sqrt{S}=7 \mathrm{TeV}$ (cf. section 4.4).

\subsection{Parameter scan}

The impact of $\tan \beta$ and of the sbottom masses have been studied performing a parameter scan on these parameters. In this scan, the soft breaking parameters $M_{L}, M_{\tilde{b}_{R}}$, and $M_{\tilde{t}_{R}}$ appearing the squared mass matrix, eq. (A.2), are set to a common value $m_{\text {squark }}$. All other parameters are set to their SPS4 values. The scans presented in this section are obtained for three different values of $m_{\text {squark }}=\{300,600,900\} \mathrm{GeV} \cdot \tan \beta$ is varied from 10 to 50 .

In all scenarios considered, we have verified the smallness of the bottom-initiated treelevel contributions, justifying our procedure of neglecting the $\mathcal{O}\left(\alpha_{s}^{2} \alpha\right)$ contributions to this 

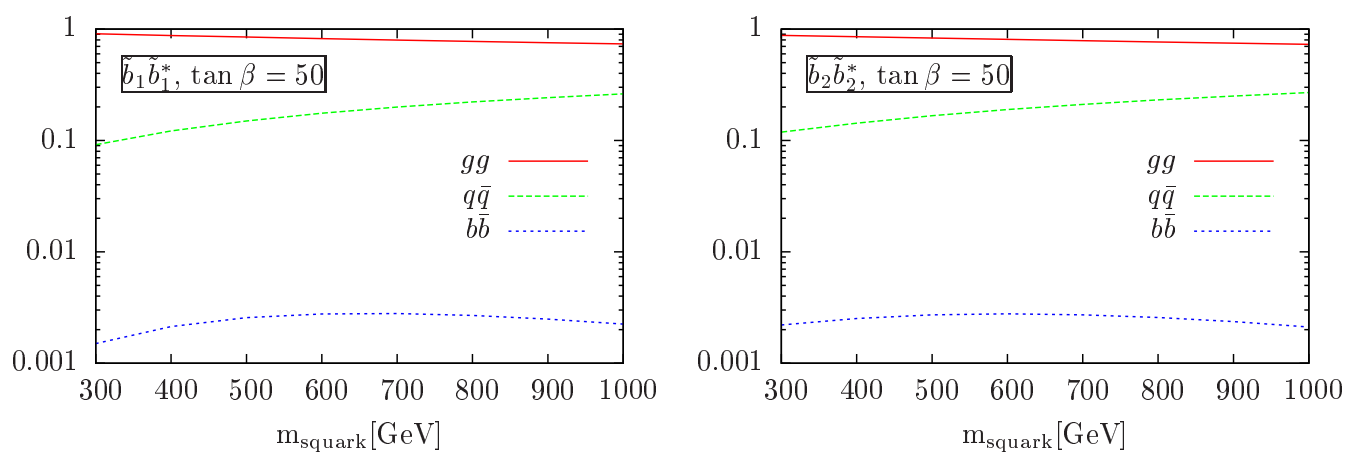

Figure 1. Relative yield of the various tree-level production channels as a function of the common squark mass breaking parameter $m_{\text {squark }}$. The left (right) plot shows $\tilde{b}_{1} \tilde{b}_{1}^{*}\left(\tilde{b}_{2} \tilde{b}_{2}^{*}\right)$ production.

channel. In figure 1 we show the relevance of the various production channels at tree-level. For $\tilde{b}_{1} \tilde{b}_{1}^{*}$ and $\tilde{b}_{2} \tilde{b}_{2}^{*}$ production the gluon fusion channel contributes $70-90 \%$ of the total cross section. The remaining $10-30 \%$ of the total cross section is given by the $q \bar{q}$ channel, its relative yield increasing with $\tan \beta$. The contribution of the $b \bar{b}$ channel is at the permille level, in accordance with the analysis in ref. [21]. ${ }^{3}$ Owing to the small yield of the $b \bar{b}$ channel at tree-level, we will safely neglect the NLO EW contributions to this channel.

The reliability of the renormalization scheme in the scenario considered has been verified by investigating the behavior of the dependent parameters since these can potentially get large finite shifts by their renormalization constants. In the case of $m_{\tilde{b}_{1}}^{2}$ and $A_{\tilde{t}}$ we have checked that the finite part of their renormalization constant is smaller than the parameter itself. The mixing angle renormalization constant enters only the counterterm of the $\tilde{b}_{1}^{*} \tilde{b}_{2}$ and $\tilde{b}_{2}^{*} \tilde{b}_{1}$ quadratic terms via the combination

$$
\delta Y_{b} \equiv\left(m_{\tilde{b}_{2}}^{2}-m_{\tilde{b}_{1}}^{2}\right) \delta \theta_{b}
$$

We have checked that the finite part of $\delta Y_{b}$ is smaller than the mass of the bottom squarks. Figure 2 shows the ratios

$$
r_{1}=\frac{\delta m_{\tilde{b}_{1}}^{2 \text { fin }}}{m_{\tilde{b}_{1}}^{2}}, \quad r_{2}=\frac{\delta A_{\tilde{t}}^{\text {fin }}}{A_{\tilde{t}}}, \quad r_{3}=\frac{\delta Y_{b}^{\text {fin }}}{m_{\tilde{b}_{1}}^{2}},
$$

as a function of $\tan \beta$ for the various values of $m_{\text {squark }}$. The value of $r_{1}, r_{2}$, and $r_{3}$ is below 0.2 in all the scenarios.

We have explicitly checked the impact of the $\mathcal{O}\left(\alpha_{s} \alpha+\alpha^{2}\right)$ contributions arising from the diagram depicted in figure 10(d). In the region of the parameter space considered in this paper these contributions are negligible. Indeed its numerical value is six orders of

\footnotetext{
${ }^{3}$ The big contributions from the $b \bar{b}$ channel quoted in ref. [27] are a consequence of two enhancement factors. First of all, the Higgs exchanged in the $s$-channel is resonant. Moreover, the $\mathcal{H} b b$ Yukawa couplings are enhanced by the choice of a negative value for the parameter $\mu$, such that $\Delta m_{b} \approx-0.76$. In our analysis the Higgs bosons are not resonant and we do not consider negative values of $\mu$, which are disfavored by the measured value of the anomalous magnetic moment of the muon $(g-2)_{\mu}[53]$.
} 


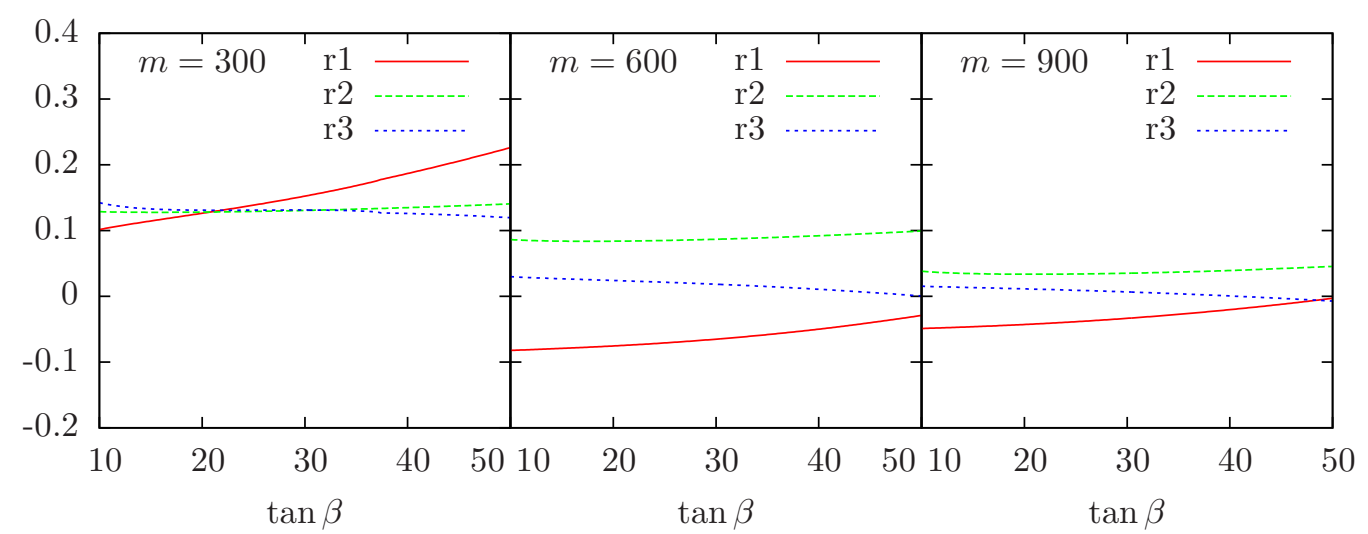

Figure 2. The ratios $r_{1}, r_{2}$, and $r_{3}$ as defined in eq. (4.5) as a function of $\tan \beta$ for different values of the common squark mass breaking parameter $m=m_{\text {squark }}$ (in $\mathrm{GeV}$ ).

magnitudes smaller than the Born cross section, justifying the approximation of identifying the CKM matrix with the unit matrix.

The results of the scan are collected in figure 3. The $q \bar{q}$ contribution is the sum of the tree-level EW and of the NLO EW contributions from the $q \bar{q}$ annihilation channel. The peaks in the corrections correspond to neutralino, chargino, or sfermion thresholds. These unphysical singularities affect the self-energy of the produced sbottom and can be regularized by taking into account the finite widths of the unstable particle [54]. The curves in figure 3 exhibit a step-function like behavior in the region where the mass of the produced sbottom is above threshold. Both, for $\tilde{b}_{1} \tilde{b}_{1}^{*}$ and for $\tilde{b}_{2} \tilde{b}_{2}^{*}$ production the behavior of the various contributions strongly depend on the size of $\tan \beta$. In the following we will distinguish between the low and the high $\tan \beta$ region, which are separated by the threshold.

The case of $\tilde{b}_{1} \tilde{b}_{1}^{*}$ production is shown in the left panels of figure 3 . The EW contributions of the $q \bar{q}$ and the $g g$ channel have substantially the same $\tan \beta$ dependence close to threshold. In the low $\tan \beta$ region the contributions from the $g \gamma$ channel cancel against the one coming from the $q \bar{q}$ annihilation. The overall effect of the EW contribution is below $1 \%$ of the tree-level QCD contribution. In the high $\tan \beta$ region the leading contributions come from the $g g$ channel and are only partly canceled by the other channels. The EW contributions are of the order of a few percents. In the $m_{\text {squark }}=900 \mathrm{GeV}$ case partial cancellations between the $g \gamma$ and the $q \bar{q}$ channels further suppress the EW contributions. In this case they are below $1 \%$. The EW contributions to $\tilde{b}_{2} \tilde{b}_{2}^{*}$ production are depicted in the right panels of figure 3 and exhibit similar features. The NLO EW contributions are more pronounced since the corrections from the $g g$ channel are more important. They are of the order of several percents, e.g. $5 \%$ for $m_{\text {squark }}=600 \mathrm{GeV}$ and $\tan \beta \geq 45$.

It is worth noticing that the only practical effect of the resummation in the $b / \tilde{b}$ sector is to change the value of the mass of the bottom squark. Indeed the impact of the effective Yukawa couplings in the computation of the amplitudes is negligible. This can be inferred from figure 4 , where we plot

$$
\delta \sigma \equiv \frac{\Delta \sigma_{\text {eff }}^{\mathrm{EW}}-\Delta \sigma_{\text {no-eff }}^{\mathrm{EW}}}{\Delta \sigma_{\text {eff }}^{\mathrm{EW}}}
$$



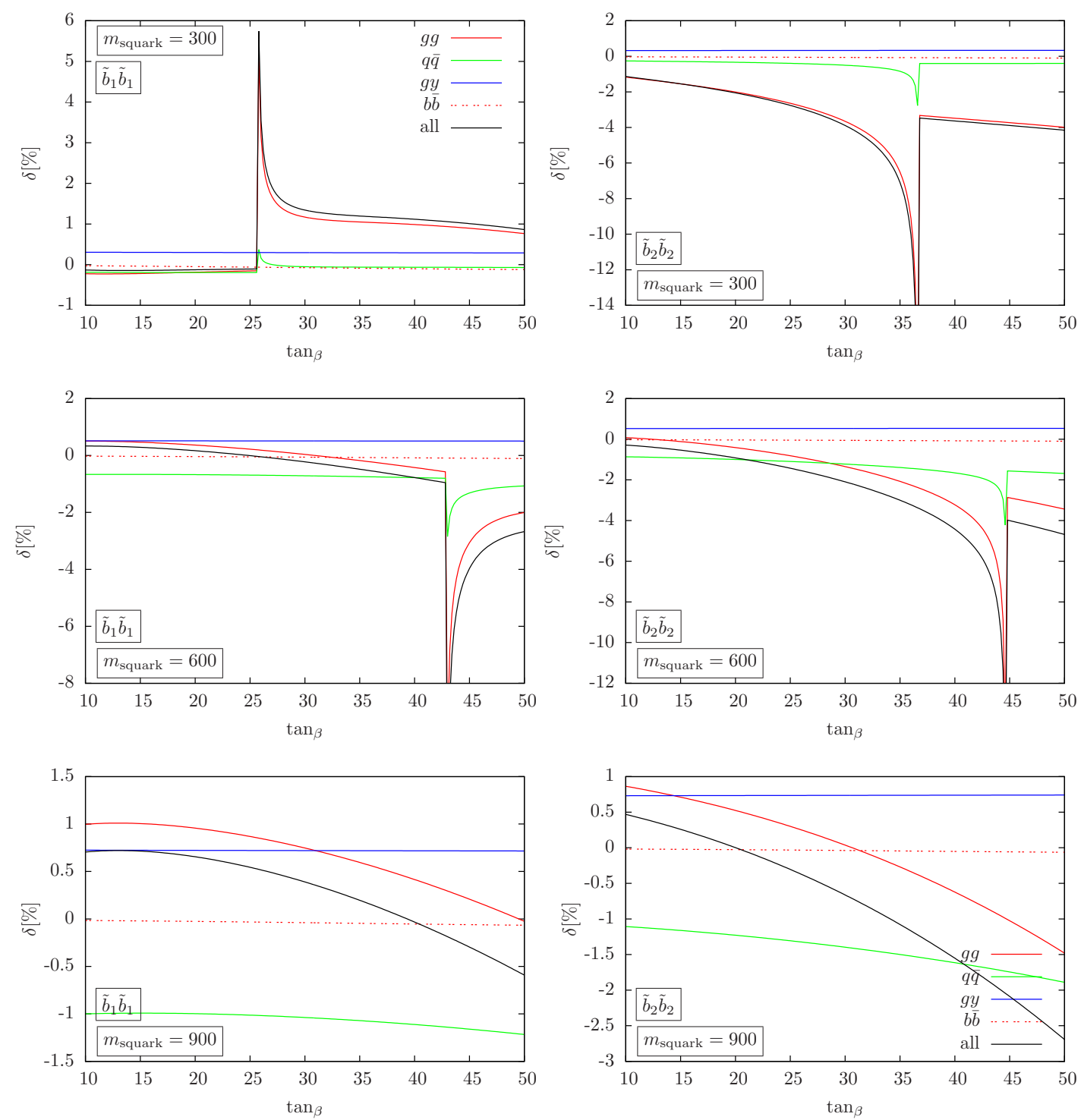

Figure 3. Relative yield of the EW contribution for $\tilde{b}_{1} \tilde{b}_{1}^{*}$ production (left panels) and for $\tilde{b}_{2} \tilde{b}_{2}^{*}$ production (right panels) as a function of $\tan \beta$. Shown are the various production channels as well as the combinded effect. The soft breaking parameter $m_{\text {squark }}$ is defined according to section 4.3 and is expressed in $\mathrm{GeV} \cdot \tan \beta$ is varied between 10 and 50 .

in the case of $\tilde{b}_{2} \tilde{b}_{2}^{*}$ production. $\Delta \sigma_{\text {eff }}^{\mathrm{EW}}$ and $\Delta \sigma_{\text {no-eff }}^{\mathrm{EW}}$ denote the EW contributions with and without the effective Yukawa couplings, respectively. The overall effect of the effective Yukawa coupling, given by the black line, is in most regions below the permille level. The positive peaks for $m_{\text {squark }}=600 \mathrm{GeV}$ and $m_{\text {squark }}=900 \mathrm{GeV}$ correspond to the zeros of the denominator in the definition (4.6), cf. figure 3.

\subsection{Differential distributions}

Even though the EW contributions seem to have a rather small impact on the total cross section, they can become important in specific phase-space regions. In figure 5 and 6 we 


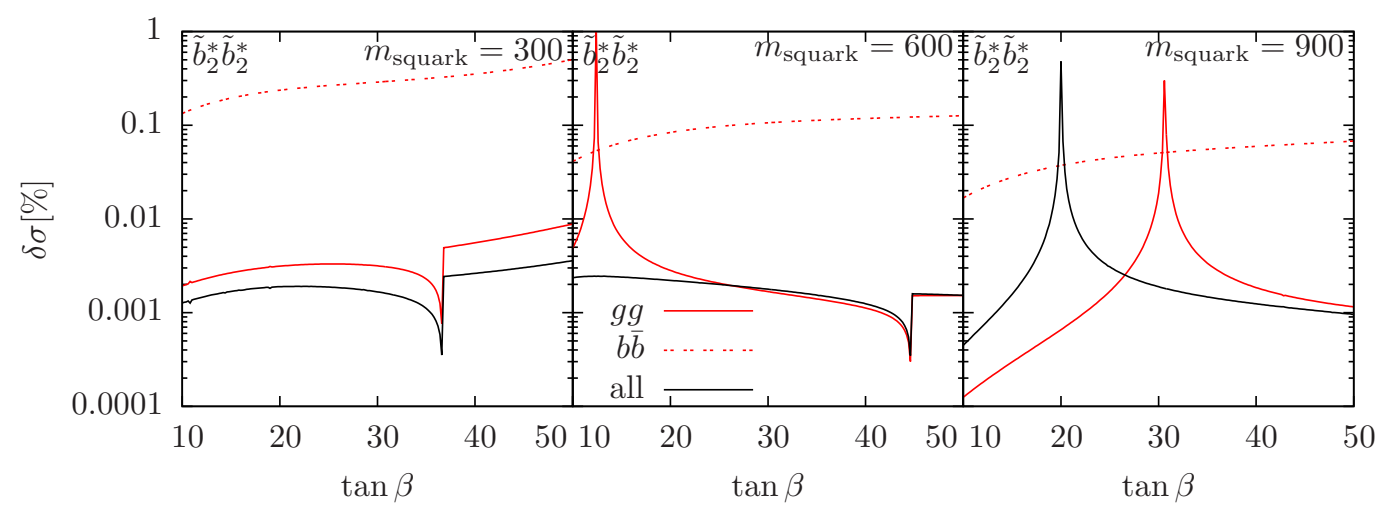

Figure 4. Relative difference between the EW contribution to the cross section for $\tilde{b}_{2} \tilde{b}_{2}^{*}$ production with and without enhanced Yukawa couplings (black lines). The individual effect on the $g g(b \bar{b})$ channel is depicted by the red(-dotted) lines. The $q \bar{q}$ channel is not affected by the resummation and hence not shown. $m_{\text {squark }}$ is given in $\mathrm{GeV}$.

consider differential distributions with respect to three different kinematical variables in the case of $\tilde{b}_{1} \tilde{b}_{1}^{*}$ and $\tilde{b}_{2} \tilde{b}_{2}^{*}$ production, respectively. We focus on the the SPS1a' scenario and on the $14 \mathrm{TeV}$ LHC. The left panels show the total EW contributions to the differential cross section. The tree-level EW contribution and the NLO EW contribution of the various production channels are depicted as well. The right panels show the impact of the EW contributions relative to the tree-level QCD cross section for each production channel. In contrast to the left panels, in the right panels the $q \bar{q}$ contribution is given by the sum of the tree-level and NLO EW contributions.

Figure 5(a) refers to the transverse momentum distribution of the sbottom with highest $p_{T}$. Close to the threshold, i.e. in the region $p_{T}<300 \mathrm{GeV}$, the contribution of the $g g$ channel is positive. Far from the threshold, this contribution becomes negative and relatively more important. In the low $p_{T}$ region the two contributions from the $q \bar{q}$ channel are different in sign, and their partial cancellation reduces the overall effect of this channel. In the high $p_{T}$ region the $q \bar{q}$ channel increases its importance. The photon induced channel peaks at low $p_{T}$ and it is almost proportional to the LO QCD cross section, i.e. its relative yield is constant in $p_{T}$. As expected, the $b \bar{b}$ channel is irrelevant in the whole $p_{T}$ region. The total EW contributions have a small positive yield of the order of $1-2 \%$ in the low $p_{T}$ region, while for $p_{T}>500 \mathrm{GeV}$ the cross section is altered by $5-10 \%$. It is interesting to note that a lower cut $p_{T \text { min }}$ on the transverse momentum can significantly rise the impact of the EW contributions. For instance the cut $p_{T \text { min }}=320 \mathrm{GeV}$ would discard the positive yield of the $g g$ channel in the low $p_{T}$ region. As a consequence the relative yield of the EW contribution to the total cross section would become of the order of $-3.2 \%$.

The invariant mass distribution is displayed in figure 5(b). The EW contributions exhibit the same high-energy behavior they have in the case of the $p_{T}$ distribution. In this energy region they alter the leading-order prediction up to $10 \%$. The peaks in the $g g$ channel correspond to $\tilde{b}_{2}$ and $\tilde{t}_{2}$ thresholds. Although in the low invariant mass region no remarkable cancellations occur among the various channels, the overall positive contribution is small, of the order of $2 \%$. 

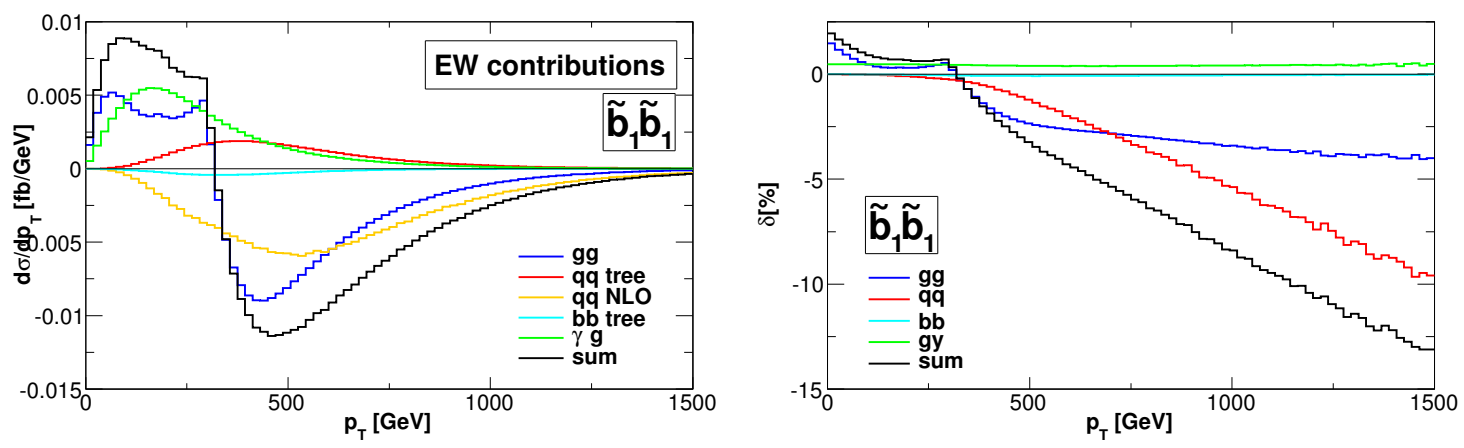

(a) $p_{T}$ distribution
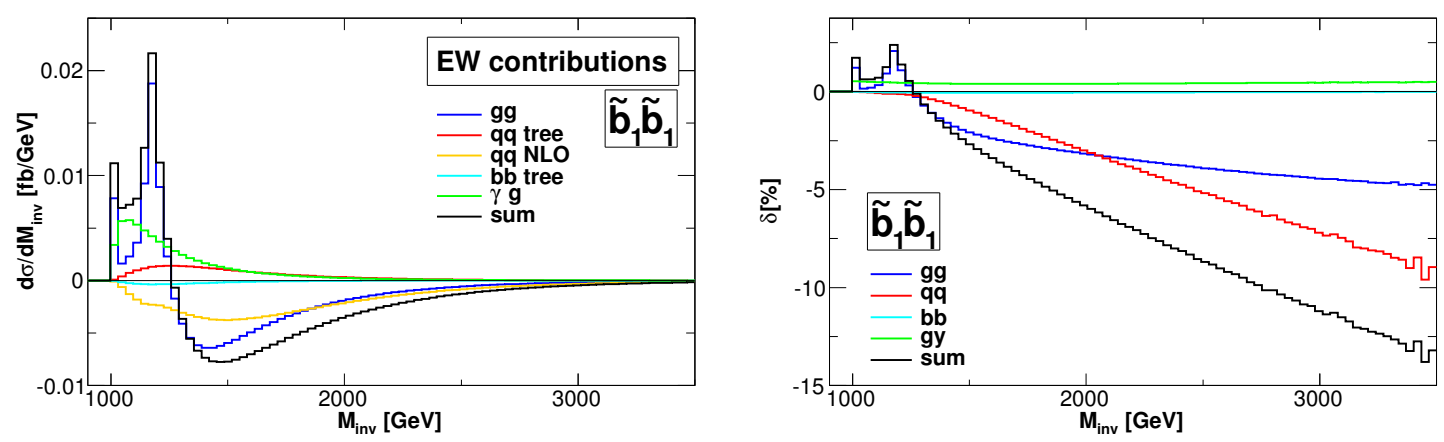

(b) $M_{\text {inv }}$ distribution
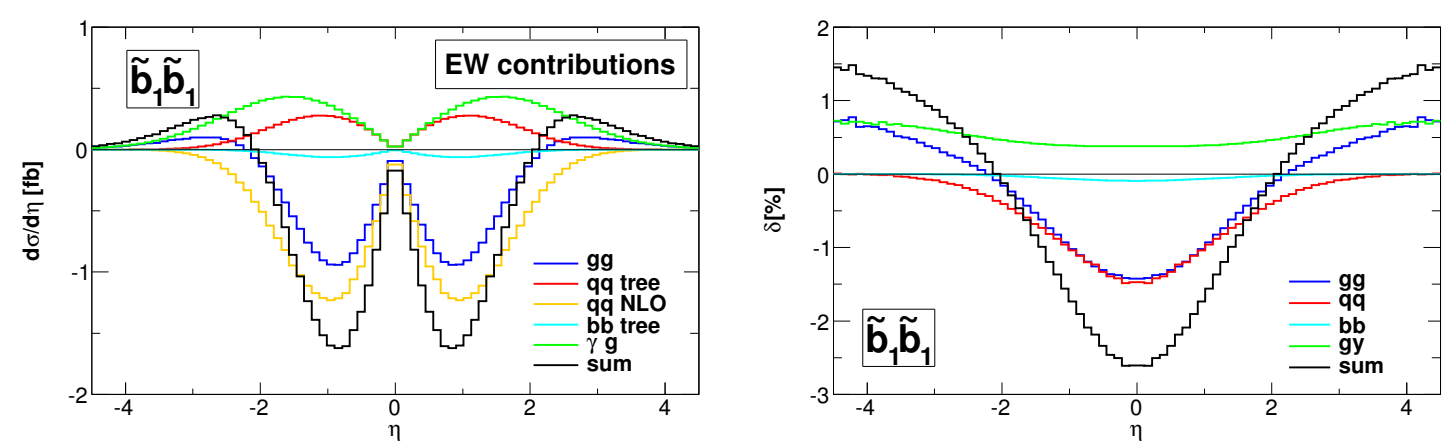

(c) $\eta$ distribution

Figure 5. Differential distributions for the transverse momentum $p_{T}$, the invariant mass $M_{\mathrm{inv}}$ and the pseudo-rapidity $\eta$ for $\tilde{b}_{1} \tilde{b}_{1}^{*}$ production at the $14 \mathrm{TeV}$ LHC within the SPS1a' scenario. Shown are the tree-level and NLO EW cross section contributions for the various production channels (left) and the impact of the NLO EW contributions relative to the LO QCD cross section (right). In the left panels the tree-level and NLO EW contributions for the $q \bar{q}$ channel are plotted separately. In the right panels they are treated inclusively.

In order to study how the EW contributions to the total cross section are altered by a lower cut $M_{\text {inv,min }}$ on the invariant mass, we consider $\sigma\left(M_{\text {inv,min }}\right)$ defined as the total cross section integrated from the value $M_{\text {inv,min }}$ of the invariant mass. The upper-left part of figure 7 shows the relative yield of the total EW contributions to $\sigma\left(M_{\mathrm{inv}, \min }\right)$, 
together with the breakdown into the individual channels. The lower cut $M_{\text {inv,min }}$ excludes the region where the EW contributions are positive. Therefore the EW contributions decreases as $M_{\text {inv,min }}$ increases, while their relative impact increases. For instance, in the region $M_{\text {inv,min }} \geq 1500 \mathrm{GeV}$ the relative yield of the EW contributions $\delta^{\mathrm{EW}}$ exceeds $-5 \%$. In this region, $\delta^{\mathrm{EW}}$ is amplified by a factor of seven with respect to its value in the case of the fully inclusive cross section, cf. table 3 . The cross section is reduced by a factor of five for $M_{\mathrm{inv}, \min }=1500 \mathrm{GeV}$ (upper-right panel of figure 7 ).

Figure 5(c) shows the pseudo-rapidity distribution, where always the squark with the higher absolute value of the pseudo-rapidity $\eta$ is considered. The gap for zero rapidity is a consequence of this definition. The NLO EW contributions peak at $|\eta|=1$ and dominate the EW contribution at this region. The contribution is negative for small values of $|\eta|$ The total effect on the LO QCD cross section is up to $2 \%$.

Figure 6 shows the differential distributions for $\tilde{b}_{2} \tilde{b}_{2}^{*}$ production in the transverse momentum (a), in the invariant mass (b), and in the pseudo-rapidity (c). In contrast to $\tilde{b}_{1} \tilde{b}_{1}^{*}$ production, the threshold behavior initiated by $\tilde{t}_{2}$ is mild and hardly visible in both, the transverse momentum and the invariant mass distributions. The EW contribution is small and its relative yield stays below $5 \%$, even in the high energy region. This is expected since in the SPS1a' scenario $\tilde{b}_{2}$ is mostly right-handed. Interestingly, the contributions from the $q \bar{q}$ channel and the $g \gamma$ channel almost cancel in most parts of the phase-space. Therefore the EW contributions are well approximated by the $g g$ channel corrections. The lower panels of figure 7 show $\sigma\left(M_{\mathrm{inv}, \mathrm{min}}\right)$ in the case of $\tilde{b}_{2} \tilde{b}_{2}^{*}$ production. In this scenario the EW contributions to $\tilde{b}_{2} \tilde{b}_{2}^{*}$ production can be safely neglected for each value of $M_{\text {inv,min. Even }}$ in the case $M_{\mathrm{inv}, \min }=2 \mathrm{TeV}$ the $\mathrm{EW}$ contributions change the Born cross section only by an amount of the order of $2 \%$.

In figure 8 we consider the differential transverse momentum distributions for $\tilde{b}_{1} \tilde{b}_{1}^{*}$ production in the SPS8 scenario for $14 \mathrm{TeV}$ (upper panels) and $7 \mathrm{TeV}$ (lower panels). It is worth analyzing the EW corrections to the $g g$ channel in this scenario. The sbottom mass is heavier in the SPS8 scenario than in the SPS1a' scenario. Therefore the gluons producing a sbottom-anti-sbottom pair have a bigger typical momentum fraction $x$ in the SPS8 scenario than in the SPS1a' scenario. Since the gluon PDF falls off rapidly at high $x$ values, the negative EW contributions of the $g g$ channel in the high $p_{T}$ region are strongly suppressed in the SPS8 scenario. In the $\sqrt{S}=7 \mathrm{TeV}$ case the typical value of $x$ it is even bigger and this phenomenon is enhanced. In the SPS8 scenario the cancellation between the positive low $p_{T}$ corrections and the negative high $p_{T}$ ones is less effective. Therefore, as mentioned in section 4.2 , the overall (positive) $g g$ channel contributions get relatively enhanced in the $\sqrt{S}=7 \mathrm{TeV}$ case.

\section{Other processes leading to bottom-squark pair production}

In this section we describe the remaining processes of (1.1) leading to a pair of bottomsquarks in the final state. The processes are non-diagonal sbottom-anti-sbottom production and (anti-)sbottom-(anti-)sbottom production,

$$
P P \rightarrow \tilde{b}_{1} \tilde{b}_{2}^{*}, \tilde{b}_{2} \tilde{b}_{1}^{*} ; \quad P P \rightarrow \tilde{b}_{\alpha} \tilde{b}_{\beta}, \tilde{b}_{\alpha}^{*} \tilde{b}_{\beta}^{*} ; \quad \alpha, \beta \in\{1,2\} .
$$



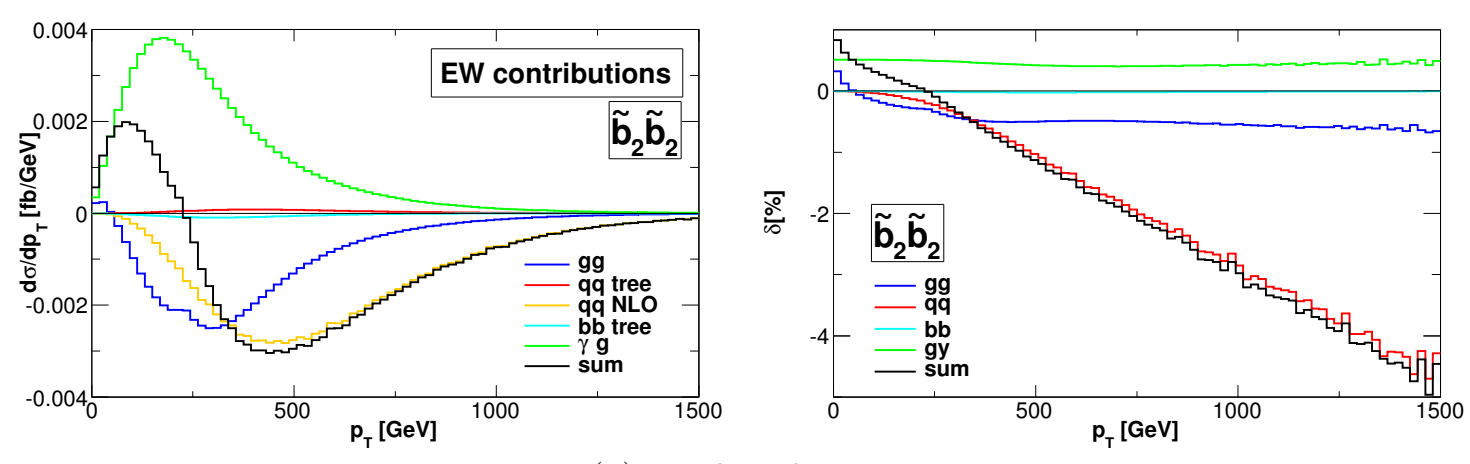

(a) $p_{T}$ distribution
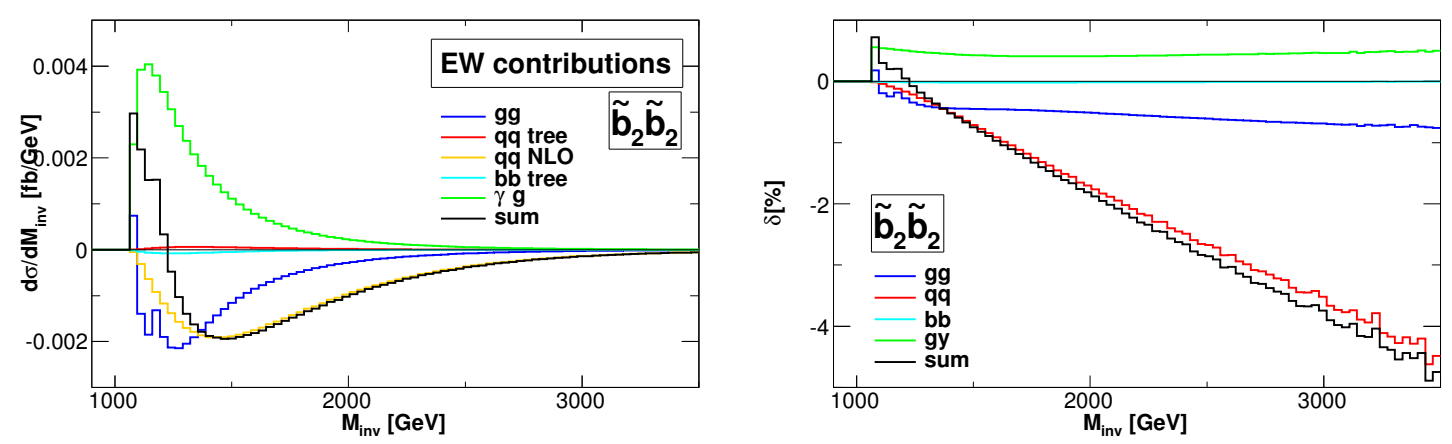

(b) $M_{\text {inv }}$ distribution
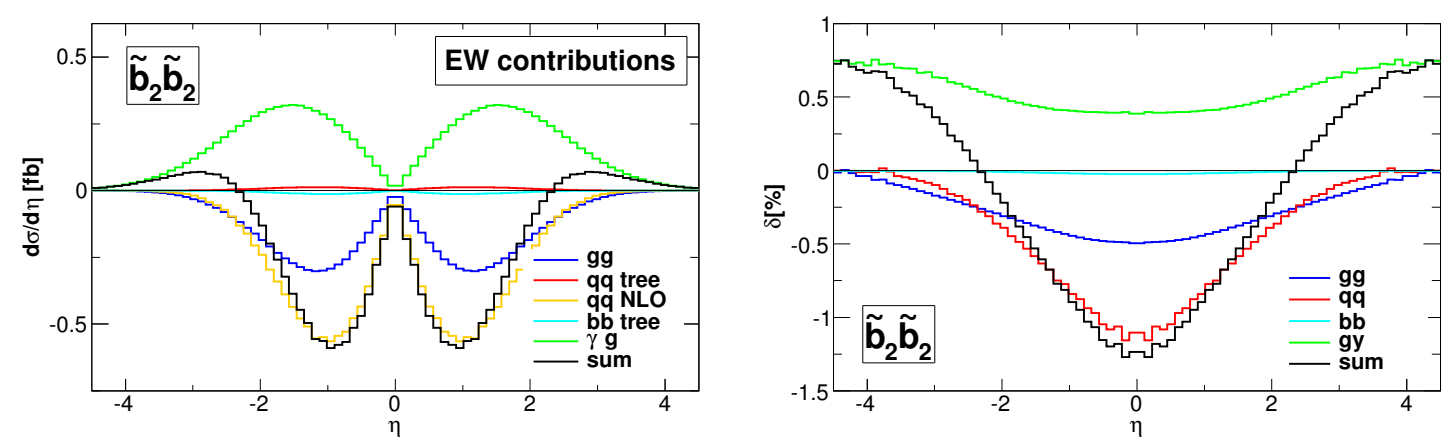

(c) $\eta$ distribution

Figure 6. Same as figure 5 but considering $\tilde{b}_{2} \tilde{b}_{2}^{*}$ production.

The leading-order cross sections, of the order $\mathcal{O}\left(\alpha_{s}^{2}\right)$, are given by

$$
\begin{aligned}
\mathrm{d} \sigma_{P P \rightarrow \tilde{b}_{1} \tilde{b}_{2}^{*}, \tilde{b}_{2} \tilde{b}_{1}^{*}}^{\mathrm{LO} Q \mathrm{D}}(S) & =\int_{\tau_{0}(1,2)}^{1} \mathrm{~d} \tau \frac{\mathrm{d} L_{b \bar{b}}}{\mathrm{~d} \tau} \mathrm{d} \hat{\sigma}_{b \bar{b} \rightarrow \tilde{b}_{1} \tilde{b}_{2}^{*}}^{2,0}(\hat{s})+\int_{\tau_{0}(1,2)}^{1} \mathrm{~d} \tau \frac{\mathrm{d} L_{b \bar{b}}}{\mathrm{~d} \tau} \mathrm{d} \hat{\sigma}_{b \bar{b} \rightarrow \tilde{b}_{2} \tilde{b}_{1}^{*}}^{2,0}(\hat{s}), \\
\mathrm{d} \sigma_{P P \rightarrow \tilde{b}_{\alpha} \tilde{b}_{\beta}, \tilde{b}_{\alpha}^{*} \tilde{b}_{\beta}^{*}}^{\mathrm{LO} Q \mathrm{CD}}(S) & =\int_{\tau_{0}(\alpha, \beta)}^{1} \mathrm{~d} \tau \frac{\mathrm{d} L_{b b}}{\mathrm{~d} \tau} \mathrm{d} \hat{\sigma}_{b b \rightarrow \tilde{b}_{\alpha} \tilde{b}_{\beta}}^{2,0}(\hat{s})+\int_{\tau_{0}(\alpha, \beta)}^{1} \mathrm{~d} \tau \frac{\mathrm{d} L_{\bar{b} \bar{b}}}{\mathrm{~d} \tau} \mathrm{d} \hat{\sigma}_{\bar{b} \bar{b} \rightarrow \tilde{b}_{\alpha}^{*} \tilde{b}_{\beta}^{*}}^{2,0}(\hat{s}),
\end{aligned}
$$



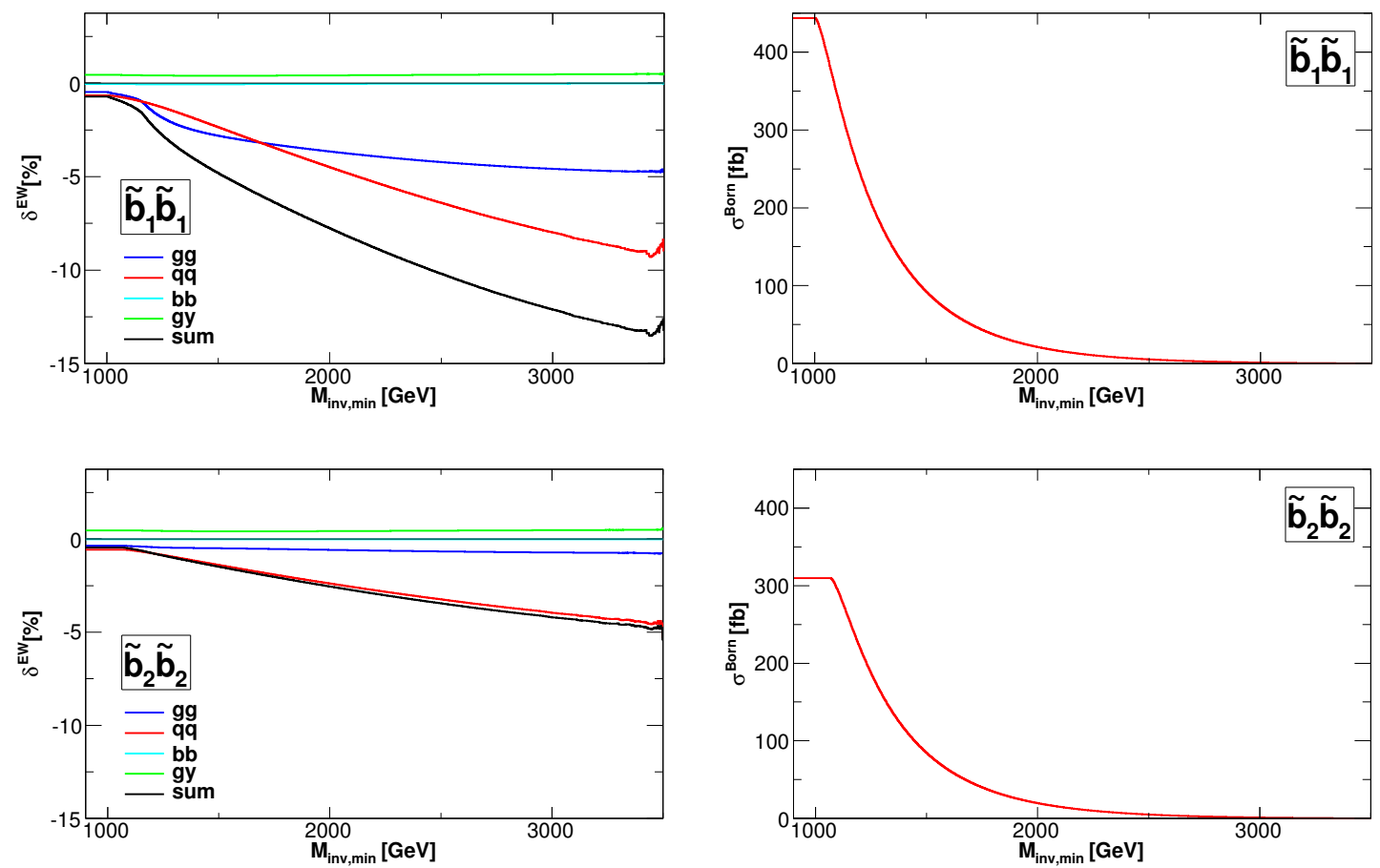

Figure 7. Left: Relative yield of the EW contributions of the different production channels in $\sigma\left(M_{\text {inv,min }}\right)$. Right: LO QCD prediction for the same observable.

where $\tau_{0}(\alpha, \beta) \equiv\left(m_{\tilde{b}_{\alpha}}+m_{\tilde{b}_{\beta}}\right)^{2} / S$ is the production threshold. The parton luminosities $\mathrm{d} L_{i j} / \mathrm{d} \tau$ are defined according to eq. (2.1). The tree-level EW contributions are of the order $\mathcal{O}\left(\alpha_{s} \alpha\right)$ and $\mathcal{O}\left(\alpha^{2}\right)$ and read as follows,

$$
\begin{aligned}
& \mathrm{d} \sigma_{P P \rightarrow \tilde{b}_{1} \tilde{b}_{2}^{*}, \tilde{b}_{2} \tilde{b}_{1}^{*}}^{\mathrm{LO} \mathrm{EW}}(S)=\int_{\tau_{0}(1,2)}^{1} \mathrm{~d} \tau \frac{\mathrm{d} L_{b \bar{b}}}{\mathrm{~d} \tau}\left[\mathrm{d} \hat{\sigma}_{b \bar{b} \rightarrow \tilde{b}_{1} \tilde{b}_{2}^{*}}^{1,1}(\hat{s})+\mathrm{d} \hat{\sigma}_{b \bar{b} \rightarrow \tilde{b}_{1} \tilde{b}_{2}^{*}}^{0,2}(\hat{s})\right] \\
& +\int_{\tau_{0}(1,2)}^{1} \mathrm{~d} \tau \frac{\mathrm{d} L_{b \bar{b}}}{\mathrm{~d} \tau}\left[\mathrm{d} \hat{\sigma}_{b \bar{b} \rightarrow \tilde{b}_{2} \tilde{b}_{1}^{*}}^{1,1}(\hat{s})+\mathrm{d} \hat{\sigma}_{b \bar{b} \rightarrow \tilde{b}_{2} \tilde{b}_{1}^{*}}^{0,2}(\hat{s})\right], \\
& \mathrm{d} \sigma_{P P \rightarrow \tilde{b}_{\alpha} \tilde{b}_{\beta}, \tilde{b}_{\alpha}^{*} \tilde{b}_{\beta}^{*}}^{\mathrm{LO} \mathrm{EW}}(S)=\int_{\tau_{0}(\alpha, \beta)}^{1} \mathrm{~d} \tau \frac{\mathrm{d} L_{b b}}{\mathrm{~d} \tau}\left[\mathrm{d} \hat{\sigma}_{b b \rightarrow \tilde{b}_{\alpha} \tilde{b}_{\beta}}^{1,1}(\hat{s})+\mathrm{d} \hat{\sigma}_{b b \rightarrow \tilde{b}_{\alpha} \tilde{b}_{\beta}}^{0,2}(\hat{s})\right] \\
& +\int_{\tau_{0}(\alpha, \beta)}^{1} \mathrm{~d} \tau \frac{\mathrm{d} L_{\bar{b} \bar{b}}}{\mathrm{~d} \tau}\left[\mathrm{d} \hat{\sigma}_{\bar{b} \bar{b} \rightarrow \tilde{b}_{\alpha}^{*} \tilde{b}_{\beta}^{*}}^{1}(\hat{s})+\mathrm{d} \hat{\sigma}_{\bar{b} \bar{b} \rightarrow \tilde{b}_{\alpha}^{*} \tilde{b}_{\beta}^{*}}^{0,}(\hat{s})\right] .
\end{aligned}
$$

The numerical impact of these processes on bottom-squark pair production at tree-level is rather small. In figure 9 we show the relative yield of diagonal sbottom-anti-sbottom production, eq. (1.2), and of all the processes described in this section as a function of the soft breaking parameter $m_{\text {squark }}$. Owing to the smallness of the (anti-)bottom PDF, the tree-level contribution of the processes (5.1) is below $1 \%$. We therefore do not include NLO EW corrections; they are expected to be even smaller and thus can be safely neglected. 

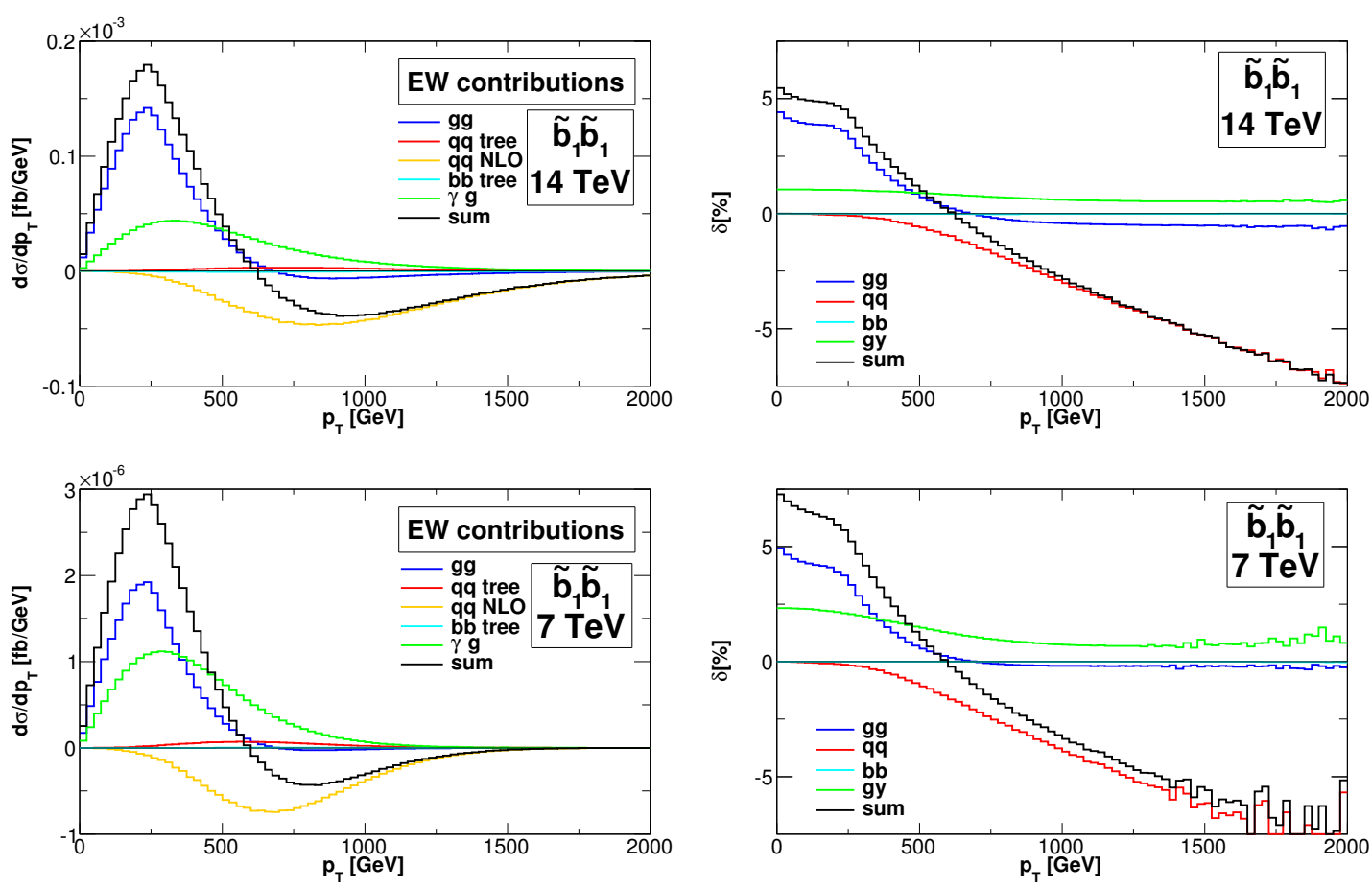

Figure 8. Differential transverse momentum distribution for $\tilde{b}_{1} \tilde{b}_{1}^{*}$ production within the SPS8 scenario for the $14 \mathrm{TeV}$ (upper plots) and $7 \mathrm{TeV}$ (lower plots) LHC.

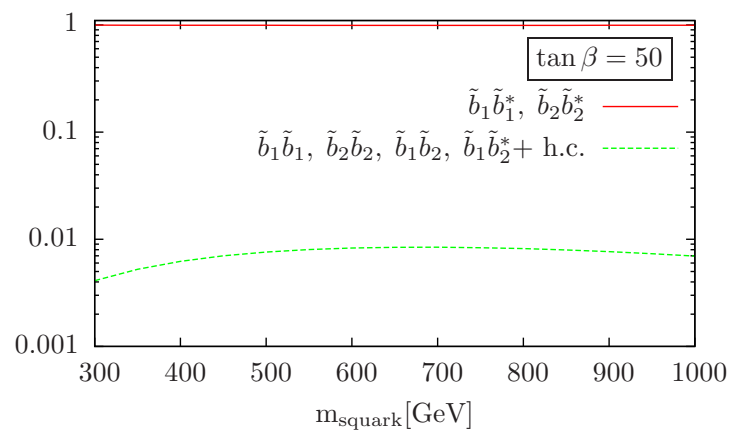

Figure 9. Relative yield of the various hadronic processes with a bottom-squark pair in the final state as a function of the common squark mass breaking parameter $m_{\text {squark }}$. The parameter $m_{\text {squark }}$ is defined in section 4.3 .

\section{Conclusions}

We have studied the EW contributions to bottom squark pair production at the LHC within the MSSM. The tree-level EW contributions from both the $q \bar{q}$-annihilation and the gluon fusion channels have been supplemented by the contribution from the photoninduced channel, not included in previous discussions. We have presented the first complete computation of the NLO EW contributions to diagonal sbottom-anti-sbottom production; together with the QCD corrections they complete the NLO analysis of the class of squarkanti-squark production processes. 
Renormalization of the $\tilde{b}$-sector is shown in detail, with a check of the reliability of the adopted renormalization scheme in the numerical analysis. The potentially large corrections to the bottom Yukawa couplings have been resummed, introducing effective couplings. In the scenarios considered, the main effect is to change the value of the masses of the bottom squarks.

The EW contributions to the total cross section are strongly scenario dependent. However, in all the scenarios considered they are of the order of few percents of the LO contribution in inclusive cross sections. Their size is partly due to strong cancellations among different channels. The EW contributions of different channels peak in different regions of the phase space. Therefore, the EW contributions are enhanced whenever kinematical cuts are applied. For similar reasons the impact of the EW contributions is more important in differential distributions, in particular in the high-energy region. In the SPS1a' scenario, and in the case of $\tilde{b}_{1} \tilde{b}_{1}^{*}$ production, they can even exceed $10 \%$ of the LO contributions for invariant mass and transverse momentum distributions.

\section{Acknowledgments}

E.M. is supported by the European Research Council under Advanced Investigator Grant ERC-AdG-228301. This work was supported in part by the European Communitys MarieCurie Research Training Network under contract MRTN-CT-2006-035505 Tools and Precision Calculations for Physics Discoveries at Colliders (HEPTOOLS).

\section{A Renormalization of the stop and sbottom sector}

In the MSSM the kinetic terms in the stop and sbottom sector read as follows

$$
\mathcal{L}=\sum_{\tilde{q}=\tilde{t}, \tilde{b}}\left\{\left(\partial_{\mu} \tilde{q}_{L}^{*}, \partial_{\mu} \tilde{q}_{R}^{*}\right)\left(\begin{array}{c}
\partial^{\mu} \tilde{q}_{L} \\
\partial^{\mu} \tilde{q}_{R}
\end{array}\right)-\left(\tilde{q}_{L}^{*}, \tilde{q}_{R}^{*}\right) \mathbf{M}_{\tilde{q}}^{2}\left(\begin{array}{c}
\tilde{q}_{L} \\
\tilde{q}_{R}
\end{array}\right)\right\}
$$

with the squared-mass matrix

$$
\mathbf{M}_{\tilde{q}}^{2}=\left(\begin{array}{cc}
M_{L}^{2}+m_{q}^{2}+m_{Z}^{2} \cos 2 \beta\left(T_{q}^{3}-e_{q} s_{\mathrm{w}}^{2}\right) & m_{q}\left(A_{\tilde{q}}-\mu \lambda_{\tilde{q}}\right) \\
m_{q}\left(A_{\tilde{q}}-\mu \lambda_{\tilde{q}}\right) & M_{\tilde{q}, R}^{2}+m_{q}^{2}+e_{q} M_{Z}^{2} \cos 2 \beta s_{\mathrm{w}}^{2}
\end{array}\right) .
$$

$M_{L}, M_{\tilde{q}, R}, A_{\tilde{q}}$ are soft breaking parameters, while $\mu$ is the supersymmetric Higgs mass parameter. $m_{q}, e_{q}, T_{q}^{3}$ are the mass, the charge and the isospin of the quark $q$, respectively. $s_{\mathrm{w}}$ is the sine of the weak mixing angle, while $\lambda_{\tilde{t}}=\cot \beta$ and $\lambda_{\tilde{b}}=\tan \beta$. The matrix (A.2) is symmetric and can be diagonalised by an orthogonal matrix $\mathbf{R}_{\tilde{q}}$ such that

$$
\left(\begin{array}{c}
\tilde{q}_{1} \\
\tilde{q}_{2}
\end{array}\right)=\mathbf{R}_{\tilde{q}}\left(\begin{array}{c}
\tilde{q}_{L} \\
\tilde{q}_{R}
\end{array}\right), \quad \mathbf{R}_{\tilde{q}}=\left(\begin{array}{cc}
R_{\tilde{q} 1,1} & R_{\tilde{q} 1,2} \\
R_{\tilde{q} 2,1} & R_{\tilde{q} 2,2}
\end{array}\right) .
$$

In the rotated basis the squared-mass matrix is diagonal

$$
\mathbf{D}_{\tilde{q}}=\mathbf{R}_{\tilde{q}} \mathbf{M}_{\tilde{q}} \mathbf{R}_{\tilde{q}}^{\top}=\left(\begin{array}{cc}
m_{\tilde{q}_{1}}^{2} & 0 \\
0 & m_{\tilde{q}_{2}}^{2}
\end{array}\right)
$$


The rotation matrix $\mathbf{R}_{\tilde{q}}$ can be parametrized in terms of a mixing angle $\theta_{\tilde{q}}$

$$
\mathbf{R}_{\tilde{q}}=\left(\begin{array}{cc}
\cos \theta_{\tilde{q}} & \sin \theta_{\tilde{q}} \\
-\sin \theta_{\tilde{q}} & \cos \theta_{\tilde{q}}
\end{array}\right) \quad \text { or } \quad \mathbf{R}_{\tilde{q}}=\left(\begin{array}{cc}
-\sin \theta_{\tilde{q}} & \cos \theta_{\tilde{q}} \\
\cos \theta_{\tilde{q}} & \sin \theta_{\tilde{q}}
\end{array}\right)
$$

depending on the sign of the determinant of $\mathbf{R}_{\tilde{q}}$. The mixing angle $\theta_{\tilde{q}}$ and the trilinear coupling $A_{\tilde{q}}$ are related via

$$
\sin 2 \theta_{\tilde{q}}=\frac{2 m_{q}\left(A_{\tilde{q}}-\mu \lambda\right)}{m_{\tilde{q}_{1}}^{2}-m_{\tilde{q}_{2}}^{2}} \xi_{\tilde{q}}, \quad \xi_{\tilde{q}} \equiv \operatorname{det}\left[\mathbf{R}_{\tilde{q}}\right]
$$

Because of SU(2)-invariance the eigenvalues of $\mathbf{M}_{\tilde{t}}^{2}$ and $\mathbf{M}_{\tilde{b}}^{2}$ are connected. In particular they have to satisfy the relation

$$
R_{\tilde{b} 1,1}^{2} m_{\tilde{b}_{1}}^{2}+R_{\tilde{b} 2,1}^{2} m_{\tilde{b}_{2}}^{2}-m_{b}^{2}=R_{\tilde{t} 1,1}^{2} m_{\tilde{t}_{1}}^{2}+R_{\tilde{t} 2,1}^{2} m_{\tilde{t}_{2}}^{2}-m_{t}^{2}-m_{W}^{2} \cos 2 \beta .
$$

\section{Definition of the " $\overline{\mathrm{DR}}$ bottom-quark mass" renormalization scheme}

In this scheme the independent parameters are chosen to be

$$
m_{\tilde{t}_{1}}^{2}, \quad m_{\tilde{t}_{2}}^{2}, \quad m_{\tilde{b}_{2}}^{2}, \quad m_{t}, m_{b}, A_{\tilde{b}}, \theta_{\tilde{t}}
$$

The squark mass squared and the top quark mass are defined in the on-shell (OS) scheme, while the bottom quark mass and the trilinear coupling $A_{\tilde{b}}$ are fixed using the $\overline{\mathrm{DR}}$ prescription,

$$
\begin{aligned}
\delta m_{\tilde{q}_{a}}^{2}= & \operatorname{Re}\left\{\Sigma_{\tilde{q} a, a}\left(m_{\tilde{q}_{a}}^{2}\right)\right\}, \text { with } \tilde{q}_{a}=\tilde{t}_{1}, \tilde{t}_{2}, \tilde{b}_{2}, \\
\delta m_{t}= & \frac{m_{t}}{2} \operatorname{Re}\left\{\Sigma_{t L}\left(m_{t}\right)+\Sigma_{t R}\left(m_{t}\right)+2 \Sigma_{t S}\left(m_{t}\right)\right\}, \\
\delta m_{b}= & \frac{m_{b}}{2} \operatorname{Re}\left\{\Sigma_{b L}^{\operatorname{div.}}\left(m_{b}\right)+\Sigma_{b R}^{\text {div. }}\left(m_{b}\right)+2 \Sigma_{b S}^{\text {div. }}\left(m_{b}\right)\right\}, \\
\delta A_{\tilde{b}}= & \frac{1}{m_{b}}\left[\frac{R_{\tilde{b} 1,1} R_{\tilde{b} 2,2}+R_{\tilde{b} 1,2} R_{\tilde{b} 2,1}}{2}\left(\operatorname{Re}\left\{\Sigma_{\tilde{b} 1,2}^{\text {div. }}\left(m_{\tilde{b}_{2}}^{2}\right)\right\}+\operatorname{Re}\left\{\Sigma_{\tilde{b} 1,2}^{\text {div. }}\left(m_{\tilde{b}_{1}}^{2}\right)\right\}\right)\right. \\
& \left.+R_{\tilde{b} 1,1} R_{\tilde{b} 1,2}\left(\operatorname{Re}\left\{\Sigma_{\tilde{b} 1,1}^{\operatorname{div} .}\left(m_{\tilde{b}_{1}}^{2}\right)\right\}-\operatorname{Re}\left\{\Sigma_{\tilde{b} 2,2}^{\text {div. }}\left(m_{\tilde{b}_{2}}^{2}\right)\right\}\right)-\frac{A_{\tilde{b}}-\mu \tan \beta}{2} \delta m_{b}\right] \\
& +\delta \mu \tan \beta+\mu \delta \tan \beta .
\end{aligned}
$$

$\delta \mu$ and $\delta \tan \beta$ appearing in eq. (A.6) are defined in the $\overline{\mathrm{DR}}$ scheme. $\Sigma^{\text {div. }}$ is the divergent part of the scalar self energies defined according to the following Lorentz decomposition,

$$
\Sigma_{q}(p)=\not p \omega_{-} \Sigma_{q L}(p)+\not p \omega_{+} \Sigma_{q R}(p)+m_{q} \Sigma_{q S}(p) .
$$

The stop mixing angle is defined according to

$$
\delta \theta_{\tilde{t}}=\frac{\xi_{\tilde{t}}\left(\operatorname{Re}\left\{\Sigma_{\tilde{t} 1,2}\left(m_{\tilde{t}_{1}}^{2}\right)\right\}-\operatorname{Re}\left\{\Sigma_{\tilde{t} 1,2}\left(m_{\tilde{t}_{2}}^{2}\right)\right\}\right)}{2\left(m_{\tilde{t}_{1}}^{2}-m_{\tilde{t}_{2}}^{2}\right)} .
$$


In the " $\overline{\mathrm{DR}}$ bottom-quark mass" scheme $A_{\tilde{t}}, \theta_{\tilde{b}}$ and $m_{\tilde{b}_{1}}^{2}$ are dependent quantities. Their counterterms read as follows

$$
\begin{aligned}
\delta A_{\tilde{t}}= & \frac{1}{m_{t}}\left[R_{\tilde{t} 1,1} R_{\tilde{t} 1,2}\left(\delta m_{\tilde{t}_{1}}^{2}-\delta m_{\tilde{t}_{2}}^{2}\right)+\xi_{\tilde{t}}\left(R_{\tilde{t} 1,1} R_{\tilde{t} 2,2}-R_{\tilde{t} 1,2} R_{\tilde{t} 2,1}\right) \delta \theta_{\tilde{t}}\left(m_{\tilde{t}_{1}}^{2}-m_{\tilde{t}_{2}}^{2}\right)\right. \\
& \left.-\left(A_{\tilde{t}}-\mu \cot \beta\right) \delta m_{t}\right]+\delta \mu \cot \beta-\mu \cot ^{2} \beta \delta \tan \beta \\
\delta m_{\tilde{b}_{1}}^{2}= & \frac{1}{R_{\tilde{b} 1,1}^{2}}\left[( 1 - 2 R _ { \tilde { b } 1 , 2 } ^ { 2 } ) \left(R_{\tilde{t} 1,1}^{2} \delta m_{\tilde{t}_{1}}^{2}+R_{\tilde{t} 1,2}^{2} \delta m_{\tilde{t}_{2}}^{2}-2 \xi_{\tilde{t}} R_{\tilde{t} 1,2}^{2} R_{\tilde{t} 2,2}^{2}\left(m_{\tilde{t}_{1}}^{2}-m_{\tilde{t}_{2}}^{2}\right) \delta \theta_{\tilde{t}}\right.\right. \\
& \left.-2 m_{t} \delta m_{t}-\delta m_{W}^{2} \cos 2 \beta-m_{W}^{2} \delta \cos 2 \beta\right)+R_{\tilde{b} 1,2}^{2} \delta m_{\tilde{b}_{2}}^{2} \\
& +2 R_{\tilde{b} 1,1} R_{\tilde{b} 1,2}\left(\delta A_{\tilde{b}}-\delta \mu \tan \beta-\mu \delta \tan \beta\right) \\
& \left.+\delta m_{b}\left(2 R_{\tilde{b} 1,1} R_{\tilde{b} 1,2}\left(A_{\tilde{b}}-\mu \tan \beta\right)+2\left(1-2 R_{\tilde{b} 1,2}^{2}\right) m_{b}\right)\right] \\
\delta \theta_{\tilde{b}}= & \frac{\xi_{\tilde{b}}\left(m_{\tilde{b}_{1}}^{2}-m_{\tilde{b}_{2}}^{2}\right)^{-1}}{R_{\tilde{b} 1,1} R_{\tilde{b} 2,2}+R_{\tilde{b} 1,2} R_{\tilde{b} 2,1}}\left[R_{\tilde{b} 1,1} R_{\tilde{b} 1,2}\left(\delta m_{\tilde{b}_{2}}^{2}-\delta m_{\tilde{b}_{1}}^{2}\right)+\left(A_{\tilde{b}}-\mu \tan \beta\right) \delta m_{b}\right. \\
& \left.+m_{b}\left(\delta A_{\tilde{b}}-\mu \delta \tan \beta-\delta \mu \tan \beta\right)\right] .
\end{aligned}
$$

The mass of the $W$ boson, $m_{W}$, is renormalized on-shell. In order to get finite Green functions, we also need the wavefunction renormalization of the bottom and sbottom,

$$
\begin{aligned}
\left(\begin{array}{c}
\tilde{q}_{1}^{\text {bare }} \\
\tilde{q}_{2}^{\text {bare }}
\end{array}\right) & =\left(\mathbf{1}+\frac{\delta \mathbf{Z}_{\tilde{q}}}{2}\right)\left(\begin{array}{c}
\tilde{q}_{1}^{\text {ren }} \\
\tilde{q}_{2}^{\text {ren }}
\end{array}\right), \quad \delta \mathbf{Z}_{\tilde{q}}=\left(\begin{array}{c}
\delta Z_{\tilde{q} 1,1} \delta Z_{\tilde{q} 1,2} \\
\delta Z_{\tilde{q} 2,1} \delta Z_{\tilde{q} 2,2}
\end{array}\right), \\
\omega_{ \pm} q^{\text {bare }} & =\omega_{ \pm}\left(1+\frac{1}{2} \delta Z_{q R / L}\right) q^{\text {ren }} .
\end{aligned}
$$

In the case of $\tilde{b} \tilde{b}^{*}$ production only the diagonal entries of the matrix $\delta \mathbf{Z}_{\tilde{q}}$ are needed. They are defined as

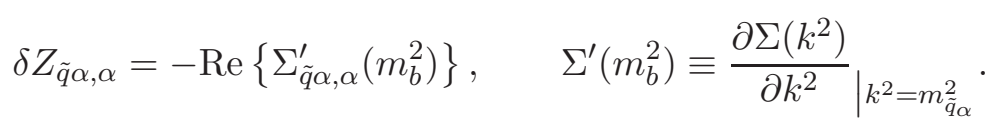

The wavefunction renormalization constants of the bottom-quark read as follows

$$
\delta Z_{b L / R}=-\operatorname{Re}\left\{\Sigma_{b L / R}\left(m_{b}^{2}\right)+m_{b}^{2}\left(\Sigma_{b L}^{\prime}\left(m_{b}^{2}\right)+\Sigma_{b R}^{\prime}\left(m_{b}^{2}\right)+2 \Sigma_{b S}^{\prime}\left(m_{b}^{2}\right)\right)\right\}
$$

In the processes considered, $\tilde{b}_{1}$ is an external particle and its mass has to be defined on-shell. Therefore we set the value of the $\tilde{b}_{1}$ mass to its OS value, obtained using the following relation

$$
m_{\tilde{b}_{1}, \text { OS }}^{2}=m_{\tilde{b}_{1}}^{2}+\delta m_{\tilde{b}_{1}}^{2}-\operatorname{Re}\left\{\Sigma_{\tilde{b} 1,1}\left(m_{\tilde{b}_{1}}^{2}\right)\right\}
$$


The renormalization constant of $m_{\tilde{b}_{1}}^{2}$ enters the counter terms in the last diagram of figure 12 and it is fixed in accordance with our choice of the $\tilde{b}_{1}$ mass,

$$
\delta m_{\tilde{b}_{1}, \text { OS }}^{2}=\operatorname{Re}\left\{\Sigma_{\tilde{b} 1,1}\left(m_{\tilde{b}_{1}, \text { OS }}^{2}\right)\right\} .
$$

The renormalization constants have to be evaluated at $\mathcal{O}(\alpha)$ and enter the calculation via the counterters in figure 12 and figure 13. The explicit expressions for the counterterms are given by

$$
\begin{aligned}
& \tilde{b}_{\alpha} \ldots \tilde{b}_{\alpha} \quad=\quad \mathrm{i}\left[\left(p^{2}-m_{\tilde{b}_{\alpha}}^{2}\right) \delta Z_{\tilde{b} \alpha, \alpha}-\delta m_{\tilde{b}_{\alpha}}^{2}\right],
\end{aligned}
$$

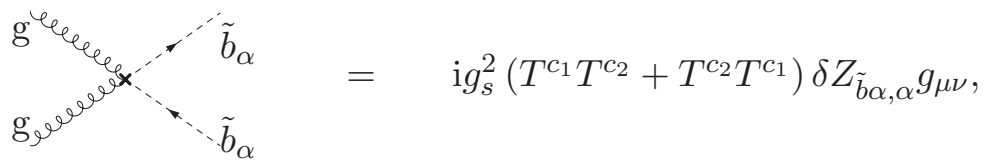

$$
\begin{aligned}
& \begin{array}{l}
g \\
\tilde{b}_{\alpha} \\
\tilde{b}_{\alpha} \\
\tilde{b}_{\alpha}
\end{array}=-\mathrm{i} g_{s} T^{c} \delta Z_{\tilde{b} \alpha, \alpha}\left(k+k^{\prime}\right)_{\mu} \text {, } \\
& g_{\bar{q}}^{q u \text { uекх }} \overbrace{\bar{q}}^{q}=-\mathrm{i} g_{s} T^{c}\left(\delta Z_{q L} \gamma_{\mu} \omega_{-}+\delta Z_{q R} \gamma_{\mu} \omega_{+}\right) \text {. }
\end{aligned}
$$

\section{B Feynman diagrams}

In this appendix we list the Feynman diagrams relevant for $\tilde{b}_{\alpha} \tilde{b}_{\alpha}^{*}$ production at tree-level and at next-to leading order electroweak. $S\left(S^{ \pm}\right)$denotes the neutral (charged) scalar Higgs and Goldstone bosons. $V=\gamma, Z$. 


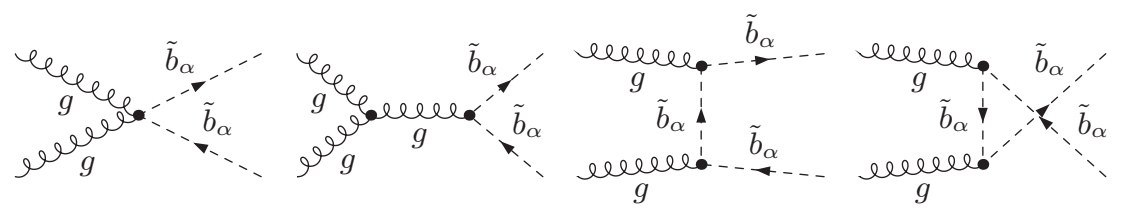

(a)

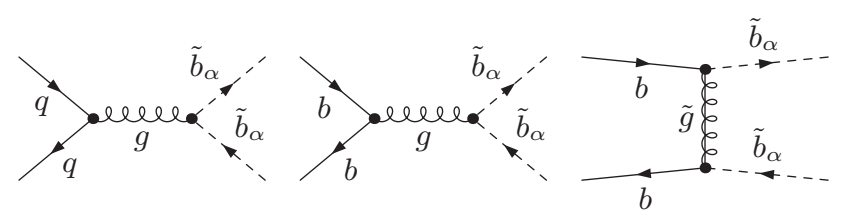

(b)

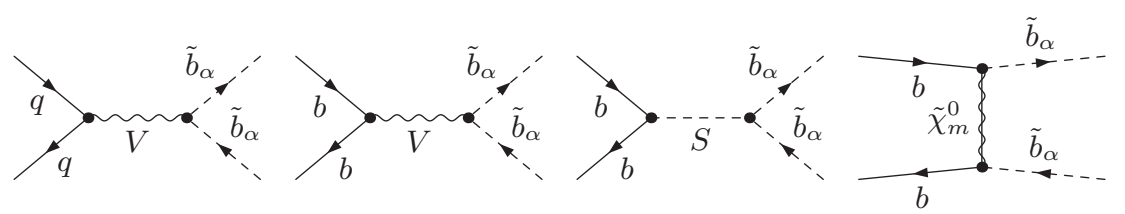

(c)

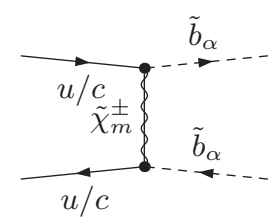

(d)

Figure 10. Tree-level Feynman diagrams for $\tilde{b}_{\alpha} \tilde{b}_{\alpha}^{*}$ production. (a) and (b) show the QCD diagrams for the $g g, q \bar{q}$ and $b \bar{b}$ channels. (c) are the EW diagrams. They are not present in the $g g$ channel at tree-level. (d) is the EW tree-level diagram involving CKM matrix. It is only present for initial $u \bar{u}$ or $c \bar{c}$ quarks.

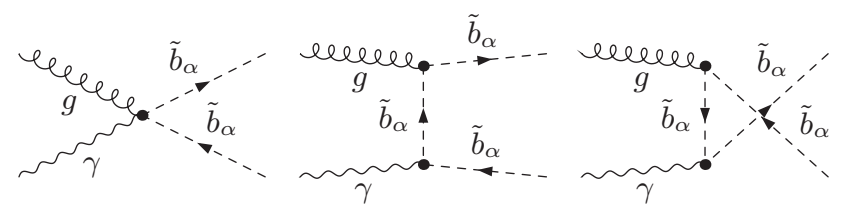

Figure 11. Lowest-order Feynman diagrams for the gluon-photon fusion process $g \gamma \rightarrow \tilde{b}_{\alpha} \tilde{b}_{\alpha}^{*}$. 


$$
\begin{aligned}
& \begin{array}{l}
\xi \xi g \\
g
\end{array}
\end{aligned}
$$

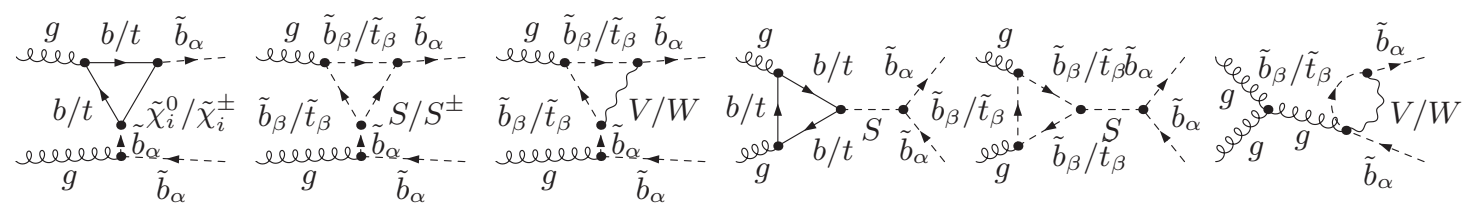

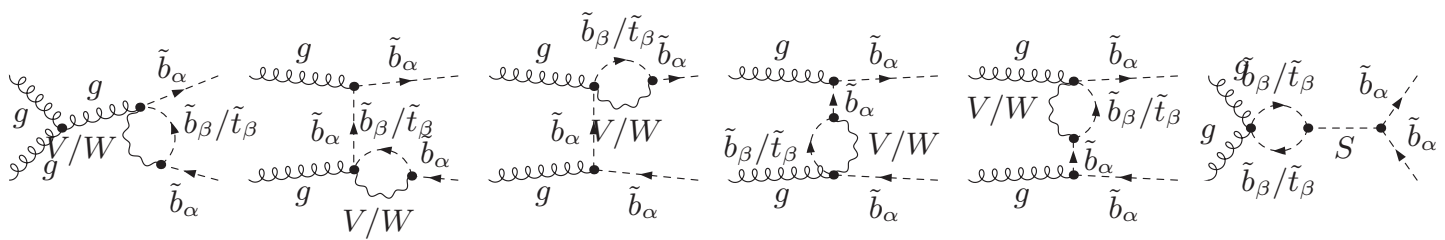

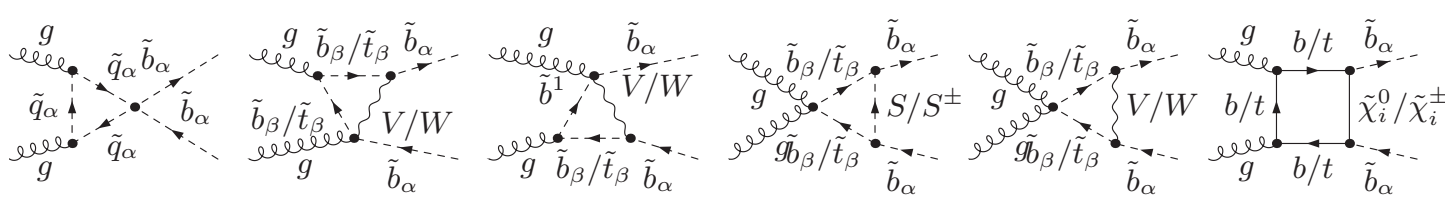

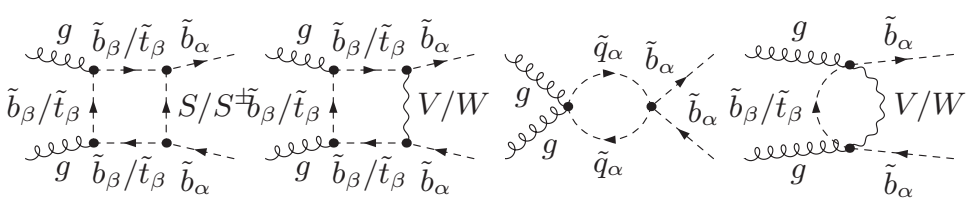

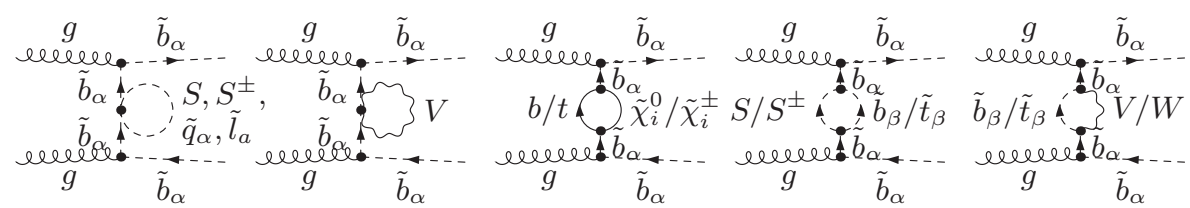

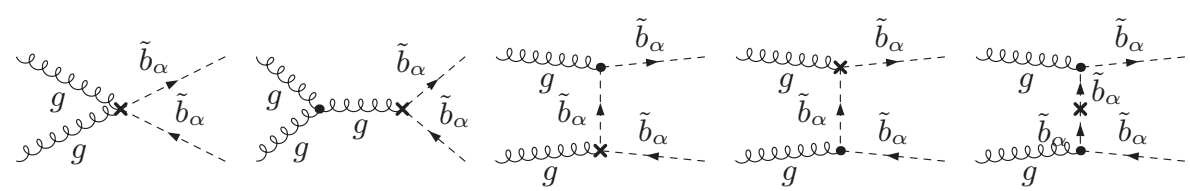

Figure 12. Virtual corrections to the process $g g \rightarrow \tilde{b}_{\alpha} \tilde{b}_{\alpha}^{*}$. A common label $V$ is used for the neutral gauge bosons $\gamma, Z^{0}$, while $S$ denotes any of the neutral Higgs bosons or the neural Goldstone boson $h^{0}, H^{0}, G^{0}$, and $S^{ \pm}$denotes the charged ones $H^{ \pm}, G^{ \pm}$. Crossed diagrams are not shown explicitly. The diagrams containing the counterterms are depicted in the last line. The counterterms have to be evaluated at $\mathcal{O}(\alpha)$. 

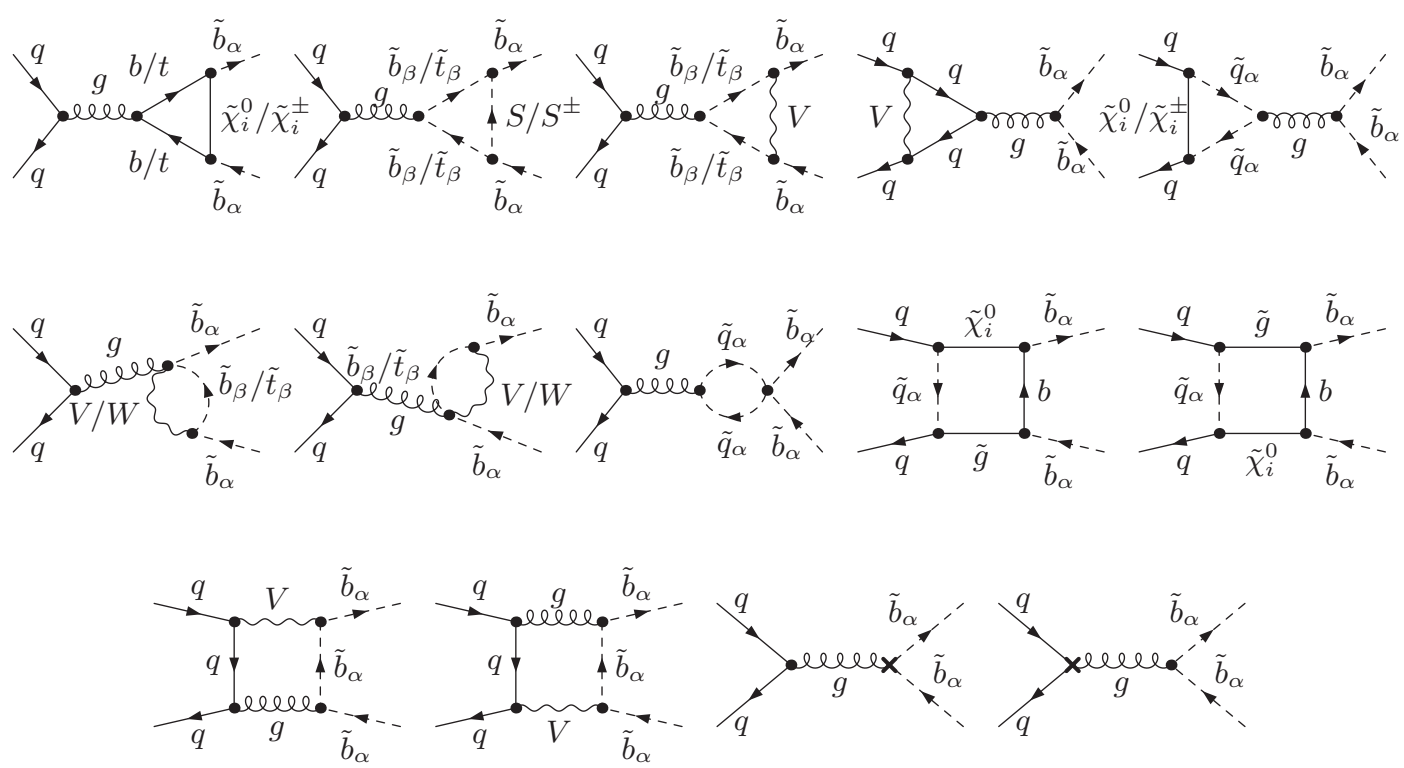

Figure 13. Virtual contributions to the process $q \bar{q} \rightarrow \tilde{b}_{\alpha} \tilde{b}_{\alpha}^{*}$. The diagrams result from EW insertions to tree-level QCD diagrams and from QCD insertions to tree-level EW diagrams. $V, S$, and $S^{ \pm}$are defined as in figure 12. Crossed diagrams are not shown. The counterterms in the last two diagrams have to be evaluated at $\mathcal{O}(\alpha)$.

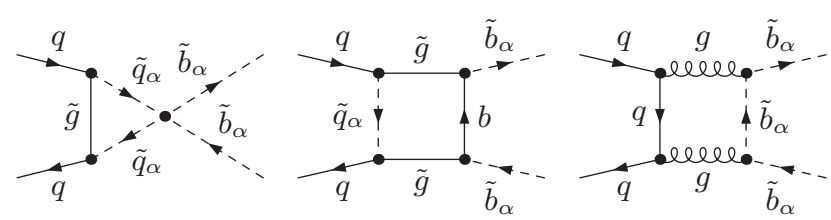

Figure 14. Virtual QCD box contributions to the process $q \bar{q} \rightarrow \tilde{b}_{\alpha} \tilde{b}_{\alpha}^{*}$. Crossed diagrams are not shown.

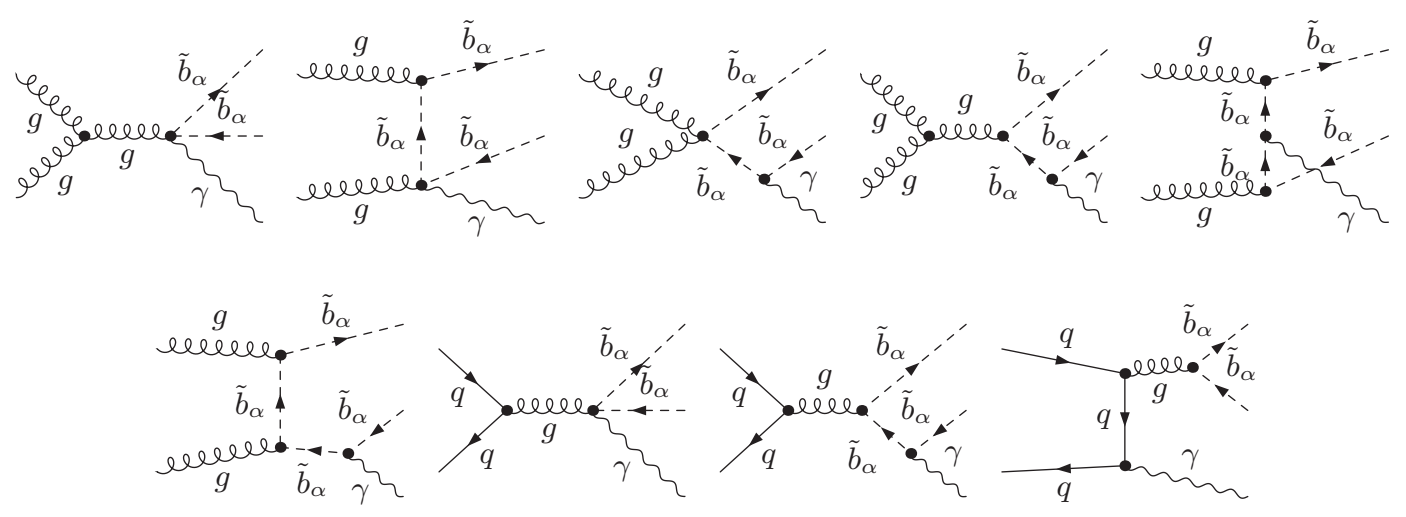

Figure 15. Real photon emission. The first six diagrams correspond to the $g g$ channel while the last three correspond to the $q \bar{q}$ channel. 


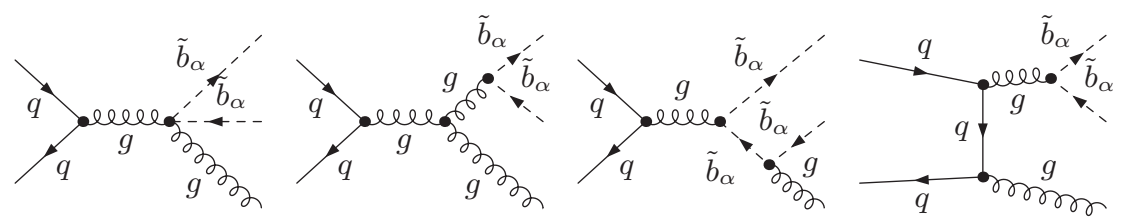

(a)

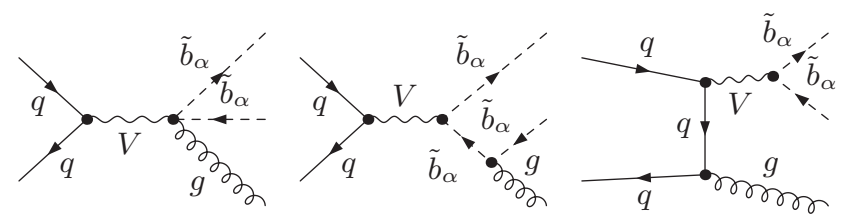

(b)

Figure 16. Real gluon emission for the $q \bar{q}$ channel. (a) QCD based diagrams. (b) EW based diagrams.
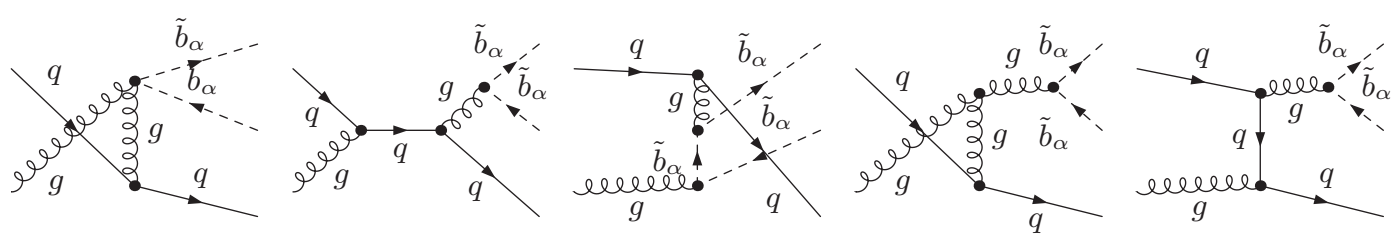

(a)
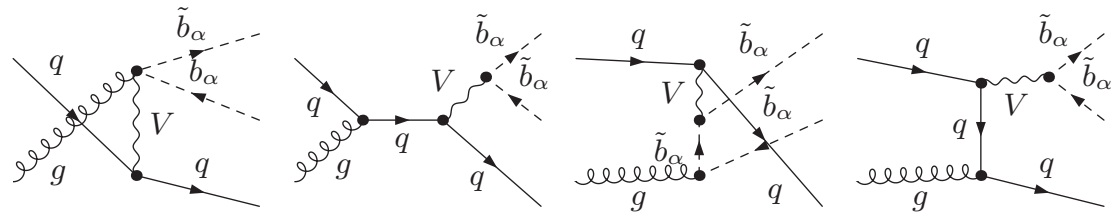

(b)

Figure 17. Feynman diagrams contributing to real quark radiation. (a) QCD based diagrams. (b) EW based diagrams. 
Open Access. This article is distributed under the terms of the Creative Commons Attribution Noncommercial License which permits any noncommercial use, distribution, and reproduction in any medium, provided the original author(s) and source are credited.

\section{References}

[1] CMS collaboration, G.L. Bayatian et al., CMS technical design report, volume II: Physics performance, J. Phys. G 34 (2007) 995 [SPIRES].

[2] ATLAS collaboration, G. Aad et al., The ATLAS Experiment at the CERN Large Hadron Collider, 2008 JINST 3 S08003 [SPIRES].

[3] ATLAS collaboration, Background studies to searches for long-lived stopped particles decaying out-of-time with LHC collisions, ATLAS report ATLAS-CONF-2010-071.

[4] ATLAS collaboration, Early supersymmetry searches with jets, missing transverse momentum and one or more leptons with the ATLAS Detector, ATLAS report ATLAS-CONF-2010-066.

[5] ATLAS collaboration, Early supersymmetry searches in channels with jets and missing transverse momentum with the ATLAS detector, ATLAS report ATLAS-CONF-2010-065.

[6] ATLAS collaboration, Early supersymmetry searches in events with missing transverse energy and b-jets with the ATLAS detector, ATLAS report ATLAS-CONF-2010-079.

[7] CMS collaboration, Performance of Methods for Data-Driven Background Estimation in SUSY Searches, CMS report, CMS PAS SUS-10-001.

[8] G.L. Kane and J.P. Leveille, Experimental Constraints on Gluino Masses and Supersymmetric Theories, Phys. Lett. B 112 (1982) 227 [SPIRES].

[9] P.R. Harrison and C.H. Llewellyn Smith, Hadroproduction of Supersymmetric Particles, Nucl. Phys. B 213 (1983) 223 [SPIRES].

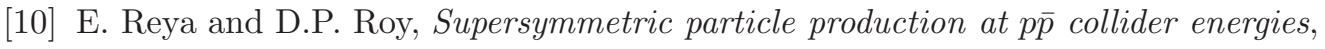
Phys. Rev. D 32 (1985) 645 [SPIRES].

[11] S. Dawson, E. Eichten and C. Quigg, Search for Supersymmetric Particles in Hadron-Hadron Collisions, Phys. Rev. D 31 (1985) 1581 [SPIRES].

[12] H. Baer and X. Tata, Component formulae for hadroproduction of left-handed and right-handed squarks, Phys. Lett. B 160 (1985) 159 [SPIRES].

[13] W. Beenakker, R. Hopker, M. Spira and P.M. Zerwas, Squark and gluino production at hadron colliders, Nucl. Phys. B 492 (1997) 51 [hep-ph/9610490] [SPIRES].

[14] W. Beenakker, M. Krämer, T. Plehn, M. Spira and P.M. Zerwas, Stop production at hadron colliders, Nucl. Phys. B 515 (1998) 3 [hep-ph/9710451] [SPIRES].

[15] W. Beenakker, R. Hopker and M. Spira, PROSPINO: A program for the PROduction of Supersymmetric Particles In Next-to-leading Order QCD, hep-ph/9611232 [SPIRES].

[16] U. Langenfeld and S.-O. Moch, Higher-order soft corrections to squark hadro-production, Phys. Lett. B 675 (2009) 210 [arXiv:0901.0802] [SPIRES].

[17] A. Kulesza and L. Motyka, Threshold resummation for squark-antisquark and gluino-pair production at the LHC, Phys. Rev. Lett. 102 (2009) 111802 [arXiv:0807.2405] [SPIRES]. 
[18] A. Kulesza and L. Motyka, Soft gluon resummation for the production of gluino-gluino and squark-antisquark pairs at the LHC, Phys. Rev. D 80 (2009) 095004 [arXiv:0905.4749] [SPIRES].

[19] M. Beneke, P. Falgari and C. Schwinn, Colour structure in threshold resummation and squark-antisquark production at NLL, PoS EPS-HEP2009 (2009) 319 [arXiv:0909.3488] [SPIRES].

[20] W. Beenakker et al., Soft-gluon resummation for squark and gluino hadroproduction, JHEP 12 (2009) 041 [arXiv:0909.4418] [SPIRES].

[21] W. Beenakker et al., Supersymmetric top and bottom squark production at hadron colliders, JHEP 08 (2010) 098 [arXiv: 1006 . 4771] [SPIRES].

[22] M. Beneke, P. Falgari and C. Schwinn, Threshold resummation for pair production of coloured heavy (s)particles at hadron colliders, Nucl. Phys. B 842 (2011) 414 [arXiv: 1007.5414] [SPIRES].

[23] W. Hollik, M. Kollar and M.K. Trenkel, Hadronic production of top-squark pairs with electroweak NLO contributions, JHEP 02 (2008) 018 [arXiv: 0712.0287] [SPIRES].

[24] S. Bornhauser, M. Drees, H.K. Dreiner and J.S. Kim, Electroweak Contributions to Squark Pair Production at the LHC, Phys. Rev. D 76 (2007) 095020 [arXiv:0709.2544] [SPIRES].

[25] W. Hollik, E. Mirabella and M.K. Trenkel, Electroweak contributions to squark-gluino production at the LHC, JHEP 02 (2009) 002 [arXiv: 0810.1044] [SPIRES].

[26] S. Bornhauser, M. Drees, H.K. Dreiner and J.S. Kim, Rapidity Gap Events in Squark Pair Production at the LHC, Phys. Rev. D 80 (2009) 095007 [arXiv:0909. 2595] [SPIRES].

[27] A. Arhrib, R. Benbrik, K. Cheung and T.-C. Yuan, Higgs boson enhancement effects on squark-pair production at the LHC, JHEP 02 (2010) 048 [arXiv: 0911.1820] [SPIRES].

[28] J. Germer, W. Hollik, E. Mirabella and M.K. Trenkel, Hadronic production of squark-squark pairs: The electroweak contributions, JHEP 08 (2010) 023 [arXiv: 1004.2621] [SPIRES].

[29] G. Bozzi, B. Fuks and M. Klasen, Non-diagonal and mixed squark production at hadron colliders, Phys. Rev. D 72 (2005) 035016 [hep-ph/0507073] [SPIRES].

[30] A.T. Alan, K. Cankocak and D.A. Demir, Squark pair production in the MSSM with explicit CP-violation, Phys. Rev. D 75 (2007) 095002 [hep-ph/0702289] [SPIRES].

[31] M. Beccaria, G. Macorini, L. Panizzi, F.M. Renard and C. Verzegnassi, Stop-antistop and sbottom-antisbottom production at LHC: a one-loop search for model parameters dependence, Int. J. Mod. Phys. A 23 (2008) 4779 [arXiv:0804.1252] [SPIRES].

[32] W. Hollik and E. Mirabella, Squark anti-squark pair production at the LHC: the electroweak contribution, JHEP 12 (2008) 087 [arXiv:0806.1433] [SPIRES].

[33] E. Mirabella, NLO electroweak contributions to gluino pair production at hadron colliders, JHEP 12 (2009) 012 [arXiv:0908.3318] [SPIRES].

[34] F.E. Paige, SUSY signatures in ATLAS at LHC, hep-ph/0307342 [SPIRES].

[35] M. Chiorboli and A. Tricomi, Squark and gluino reconstruction in CMS, CMS note, CMS-NOTE-2004-029.

[36] K. Kawagoe, M.M. Nojiri and G. Polesello, A new SUSY mass reconstruction method at the CERN LHC, Phys. Rev. D 71 (2005) 035008 [hep-ph/0410160] [SPIRES].

[37] S. Heinemeyer, W. Hollik, H. Rzehak and G. Weiglein, High-precision predictions for the MSSM Higgs sector at $\mathcal{O}\left(\alpha_{b} \alpha_{s}\right)$, Eur. Phys. J. C 39 (2005) 465 [hep-ph/0411114] [SPIRES]. 
[38] S. Heinemeyer, H. Rzehak and C. Schappacher, Proposals for Bottom Quark/Squark Renormalization in the Complex MSSM, Phys. Rev. D 82 (2010) 075010 [arXiv:1007.0689] [SPIRES].

[39] M.S. Carena, D. Garcia, U. Nierste and C.E.M. Wagner, Effective Lagrangian for the $\bar{t} b H^{+}$ interaction in the MSSM and charged Higgs phenomenology, Nucl. Phys. B 577 (2000) 88 [hep-ph/9912516] [SPIRES].

[40] T. Hahn, Generating Feynman diagrams and amplitudes with FeynArts 3, Comput. Phys. Commun. 140 (2001) 418 [hep-ph/0012260] [SPIRES].

[41] T. Hahn and M. Rauch, News from FormCalc and LoopTools, Nucl. Phys. Proc. Suppl. 157 (2006) 236 [hep-ph/0601248] [SPIRES].

[42] T. Hahn and C. Schappacher, The implementation of the minimal supersymmetric standard model in FeynArts and FormCalc, Comput. Phys. Commun. 143 (2002) 54 [hep-ph/0105349] [SPIRES].

[43] U. Baur, S. Keller and D. Wackeroth, Electroweak radiative corrections to $W$ boson production in hadronic collisions, Phys. Rev. D 59 (1999) 013002 [hep-ph/9807417] [SPIRES].

[44] L.J. Hall, R. Rattazzi and U. Sarid, The Top quark mass in supersymmetric SO(10) unification, Phys. Rev. D 50 (1994) 7048 [hep-ph/9306309] [SPIRES].

[45] R. Hempfling, Yukawa coupling unification with supersymmetric threshold corrections, Phys. Rev. D 49 (1994) 6168 [SPIRES].

[46] M.S. Carena, M. Olechowski, S. Pokorski and C.E.M. Wagner, Electroweak symmetry breaking and bottom-top Yukawa unification, Nucl. Phys. B 426 (1994) 269 [hep-ph/9402253] [SPIRES].

[47] D.M. Pierce, J.A. Bagger, K.T. Matchev and R.-j. Zhang, Precision corrections in the minimal supersymmetric standard model, Nucl. Phys. B 491 (1997) 3 [hep-ph/9606211] [SPIRES].

[48] Particle Data Group collaboration, K. Nakamura et al., Review of particle physics, J. Phys. G 37 (2010) 075021 [SPIRES].

[49] Tevatron Electroweak Working Group, CDF, D0 collaboration, Combination of CDF and D0 results on the mass of the top quark, hep-ex/0603039 [SPIRES].

[50] J.A. Aguilar-Saavedra et al., Supersymmetry parameter analysis: SPA convention and project, Eur. Phys. J. C 46 (2006) 43 [hep-ph/0511344] [SPIRES].

[51] B.C. Allanach, SOFTSUSY: a C++ program for calculating supersymmetric spectra, Comput. Phys. Commun. 143 (2002) 305 [hep-ph/0104145] [SPIRES].

[52] A.D. Martin, R.G. Roberts, W.J. Stirling and R.S. Thorne, Parton distributions incorporating QED contributions, Eur. Phys. J. C 39 (2005) 155 [hep-ph/0411040] [SPIRES].

[53] D. Stöckinger, The muon magnetic moment and supersymmetry, J. Phys. G 34 (2007) R45 [hep-ph/0609168] [SPIRES].

[54] B.A. Kniehl, C.P. Palisoc and A. Sirlin, Elimination of threshold singularities in the relation between on-shell and pole widths, Phys. Rev. D 66 (2002) 057902 [hep-ph/0205304] [SPIRES]. 